\title{
CASCADE OF PHASE SHIFTS AND CREATION OF NONLINEAR FOCAL POINTS FOR SUPERCRITICAL SEMICLASSICAL HARTREE EQUATION*
}

\author{
SATOSHI MASAKI ${ }^{\dagger}$
}

\begin{abstract}
We consider the semiclassical limit of the Hartree equation with a data causing a focusing at a point. We study the asymptotic behavior of phase function associated with the WKB approximation near the caustic when a nonlinearity is supercritical. In this case, it is known that a phase shift occurs in a neighborhood of focusing time in the case of focusing cubic nonlinear Schrödinger equation. Thanks to the smoothness of the nonlocal nonlinearities, we justify the WKBtype approximation of the solution for a data which is larger than in the previous results and is not necessarily well-prepared. We also show by an analysis of the limit hydrodynamical equaiton that, however, this WKB-type approximation breaks down before reaching the focal point: Nonlinear effects lead to the formation of singularity of the leading term of the phase function.
\end{abstract}

Key words. Nonlinear Schrödinger equation, semiclassical analysis, WKB approximation, caustics, Euler equation.

AMS subject classifications. Primary 35Q55; Secondary 35Q31

1. Introduction. This paper is devoted to the study of the semiclassical limit $\varepsilon \rightarrow 0$ for the Cauchy problem of the semiclassical nonlinear Schrödinger equation for $(t, x) \in \mathbb{R}_{+} \times \mathbb{R}^{n}$

$$
i \varepsilon \partial_{t} u^{\varepsilon}+\frac{\varepsilon^{2}}{2} \Delta u^{\varepsilon}=\varepsilon^{\alpha} N\left(u^{\varepsilon}\right), \quad \quad u_{\mid t=0}^{\varepsilon}(x)=a_{0}(x) e^{-i \frac{|x|^{2}}{2 \varepsilon}}
$$

with the nonlocal nonlinearity of Hartree type

$$
N\left(u^{\varepsilon}\right)=\lambda\left(|x|^{-\gamma} *\left|u^{\varepsilon}\right|^{2}\right) u^{\varepsilon},
$$

where $n \geqslant 3, \lambda$ is a real number, and $\gamma$ is a positive number. In this paper we consider the small-nonlinearity case $\alpha>0$. The case $\alpha=0$ is studied in $[1,9]$. In the case of linear equation $N \equiv 0$, the quadratic oscillation in the initial data causes a caustic at the origin at $t=1$. In $[4,5], \mathrm{R}$. Carles justified the general heuristics presented in [20] in the case of (1.1) with $N(y)=|y|^{\beta} y$ (see, also [7]), and two different notions of the criticality for $\alpha$ are realized. One is concerned with the nonlinear effect on the behavior far from the focal point, and the other with that near the focal point. The situation is similar in the case of Hartree equation

$$
\begin{aligned}
i \varepsilon \partial_{t} u^{\varepsilon}+\frac{\varepsilon^{2}}{2} \Delta u^{\varepsilon} & =\lambda \varepsilon^{\alpha}\left(|x|^{-\gamma} *\left|u^{\varepsilon}\right|^{2}\right) u^{\varepsilon}, \\
u_{\mid t=0}^{\varepsilon}(x) & =a_{0}(x) e^{-i \frac{|x|^{2}}{2 \varepsilon}} .
\end{aligned}
$$

(see, $[8,10,27]$ ). The two critical indices are $\alpha=1$ (far from focal point) and $\alpha=\gamma$ (near the focal point). They are completely different notions. For example, the second index $\alpha=\gamma$ depends on the shape of the nonlinearity while the first not. Using terminologies in [6], we have the following nine cases:

\footnotetext{
* Received May 29, 2009; accepted for publication November 19, 2009.

$\dagger$ Division of Mathematics, Graduate School of Information Sciences, Tohoku University, Sendai 980-8579, Japan (masaki@ims.is.tohoku.ac.jp).
} 


\begin{tabular}{c|c|c|c|} 
& $\alpha>1$ & $\alpha=1$ & $\alpha<1$ \\
\hline$\alpha>\gamma$ & linear WKB & nonlinear WKB & supercritical WKB \\
& linear caustic & linear caustic & linear caustic \\
\hline$\alpha=\gamma$ & linear WKB & nonlinear WKB & supercritical WKB \\
& nonlinear caustic & nonlinear caustic & nonlinear caustic \\
\hline$\alpha<\gamma$ & linear WKB & nonlinear WKB & supercritical WKB \\
& supercritical caustic & supercritical caustic & supercritical caustic \\
\hline
\end{tabular}

Roughly speaking, the term "linear WKB" means that, far from the caustic, the propagation of $u^{\varepsilon}$ does not involve the nonlinear effect at leading order. The term "linear caustic" means that the nonlinear effect is negligible at leading order when the solution crosses the focal point.

Now, let us be more precise about this problem with (1.3)-(1.4). Let $w^{\varepsilon}$ be the solution of the linear equation $\left(i \varepsilon \partial_{t}+\left(\varepsilon^{2} / 2\right) \Delta\right) w^{\varepsilon}=0$ with the same initial data as in (1.4). Note that $w^{\varepsilon}$ is approximated by the WKB-type approximate solution

$$
v_{\operatorname{lin}}^{\varepsilon}=\frac{1}{(1-t)^{n / 2}} a_{0}\left(\frac{x}{1-t}\right) e^{i \frac{|x|^{2}}{2 \varepsilon(t-1)}}
$$

as long as $\varepsilon /(1-t)$ is small. In the linear WKB case $\alpha>1$, it is shown in $[8,26,27]$ that the solution $u^{\varepsilon}$ is close to $w^{\varepsilon}$ (and so to $v_{\text {lin }}^{\varepsilon}$ ) before caustic. The time interval in which $u^{\varepsilon}$ is approximated by $w^{\varepsilon}$ depends on the latter critical notion $\alpha=\gamma$ : If $\alpha>\gamma$ then $u^{\varepsilon} \rightarrow w^{\varepsilon}$ as $\varepsilon \rightarrow 0$ for all $t \leqslant 1$; if $\alpha=\gamma$ then it holds for $1-t \geqslant \Lambda \varepsilon$ with a large $\Lambda$; if $\alpha<\gamma$ then it holds for $1-t \geqslant \Lambda \varepsilon^{\mu}$ with a large $\Lambda$ and some $\mu=\mu(\alpha, \gamma) \leqslant(\alpha-1) /(\gamma-1)$. In the case $\alpha \geqslant \gamma$, the asymptotic profile of the solution beyond the caustic is also given in [8]. Let us proceed to the supercritical caustic case $\alpha<\gamma$. In this case, infinitely many phase shifts occur between the time $1-t=\Lambda_{1} \varepsilon^{\frac{\alpha-1}{\gamma-1}}$, called a first boundary layer, and the time $1-t=\Lambda \varepsilon^{\alpha / \gamma}$, called a final layer. This phenomena, called cascade of phase shifts, is first shown in [6] for (1.1) with certain class of nonlinearities including the cubic nonlinearity $N(y)=|y|^{2} y$, and the asymptotic behavior of the solution is given before the final layer, $1-t \gg \varepsilon^{\alpha-1 / \gamma-1}$, in the case $(\gamma>) \alpha>1$. The similar result is proven also in the nonlinear and supercritical WKB case $\alpha \leqslant 1$, provided the initial data is a properly-modified one of the form

$$
u_{\mid t=0}^{\varepsilon}(x)=b_{0}\left(\varepsilon^{\frac{\alpha}{\gamma}}, x\right) e^{-i \frac{|x|^{2}}{2 \varepsilon}} \exp \left(i \varepsilon^{\frac{\alpha}{\gamma}-1} \phi_{0}\left(\varepsilon^{\frac{\alpha}{\gamma}}, x\right)\right),
$$

where $\left(b_{0}(t, x), \phi_{0}(t, x)\right)$ is a suitable function defined in terms of $a_{0}$. Let us call this type of data as a well-prepared data.

The aim of this paper is to give an explicit asymptotic profile of the solution of (1.3)-(1.4) (before and) on the final layer for whole $\gamma>\alpha>0$ with a not-modified data. In particular, the behavior of the solution is new in the following situations:

- On the final layer, that is, at $t=1-T^{-1} \varepsilon^{\alpha / \gamma}$ with not necessarily small $T>0$.

- $\alpha \leqslant 1$ with an initial data which is not necessarily of the form (1.6).

Moreover, it will be shown that the WKB-type approximation of the solution breaks down at some time on the final layer, that is, at $t=t_{c}:=1-\left(T^{*}\right)^{-1} \varepsilon^{\alpha / \gamma}$ for some $T^{*}>0$ for a certain class of initial data.

For our analysis, we apply the following transform introduced in [6]:

$$
u^{\varepsilon}(t, x)=\frac{1}{(1-t)^{n / 2}} \psi^{\varepsilon}\left(\frac{\varepsilon^{\frac{\alpha}{\gamma}}}{1-t}, \frac{x}{1-t}\right) \exp \left(i \frac{|x|^{2}}{2 \varepsilon(t-1)}\right) .
$$


Then, (1.3)-(1.4) becomes

$$
i \varepsilon^{1-\frac{\alpha}{\gamma}} \partial_{\tau} \psi^{\varepsilon}+\frac{\left(\varepsilon^{1-\frac{\alpha}{\gamma}}\right)^{2}}{2} \Delta_{y} \psi^{\varepsilon}=\lambda \tau^{\gamma-2}\left(|y|^{-\gamma} *\left|\psi^{\varepsilon}\right|^{2}\right) \psi^{\varepsilon}, \quad \psi_{\mid \tau=\varepsilon^{\alpha / \gamma}}^{\varepsilon}(y)=a_{0}(y),
$$

where $\tau=\varepsilon^{\alpha / \gamma} /(1-t)$ and $y=x /(1-t)$. The quadratic oscillation of the initial data is canceled out. This transformation clarifies the problem; if the nonlinear effect is weak and so if $u^{\varepsilon}$ behaves like $w^{\varepsilon}$ and $v_{\text {lin }}^{\varepsilon}$, then $\psi^{\varepsilon}$ is close to $a_{0}$; if the solution has a rapid oscillation other than $\exp \left(i|x|^{2} / 2 \varepsilon(t-1)\right)$, then $\psi^{\varepsilon}$ becomes oscillatory. We change the parameter into $h=\varepsilon^{1-\alpha / \gamma}$. The limit $\varepsilon \rightarrow 0$ is equivalent to $h \rightarrow 0$ as long as $\alpha<\gamma$. Denoting $\psi^{\varepsilon}$ by $\psi^{h}$, our problem is reduced to the limit $h \rightarrow 0$ of the solution to

$$
i h \partial_{\tau} \psi^{h}+\frac{h^{2}}{2} \Delta_{y} \psi^{h}=\lambda \tau^{\gamma-2}\left(|y|^{-\gamma} *\left|\psi^{h}\right|^{2}\right) \psi^{h}, \quad \psi_{\mid \tau=h \frac{\alpha}{\gamma-\alpha}}^{h}(y)=a_{0}(y) .
$$

Since $\tau=h^{\alpha /(\gamma-\alpha)} /(1-t)$, the correspondence between boundary layers of $t$ and $\tau$ variables is as follows:

$$
\begin{aligned}
& \text { initial time: } t=0 \quad \longleftrightarrow \tau=h^{\frac{\alpha}{\gamma-\alpha}}, \\
& \text { first layer: } t=1-\Lambda_{1} \varepsilon^{\frac{\alpha-1}{\gamma-1}} \longleftrightarrow \tau=\Lambda_{1}^{-1} h^{\frac{1}{\gamma-1}}, \\
& \text { final layer: } t=1-T^{-1} \varepsilon^{\frac{\alpha}{\gamma}} \longleftrightarrow \tau=T \text {. }
\end{aligned}
$$

Our analysis of (1.8) is based on a generalized WKB method by Gérard [15] and Grenier [18]. We apply a modified Madelung transformation

$$
\psi^{h}=a^{h} \exp \left(i \frac{\phi^{h}}{h}\right)
$$

to (1.8), where $a^{h}$ is complex valued and $\phi^{h}$ is real valued. This choice is slightly different from the usual Madelung transformation

$$
\psi^{h}=\sqrt{\rho^{h}} \exp \left(i \frac{S^{h}}{h}\right)
$$

(see [14]) which leads us to an equation of compressible fluid with the quantum pressure. It is essential that $a^{h}$ takes complex value and, therefore, $\phi^{h} \neq S^{h}$ in general. The choice (1.10) allows us to rewrite (1.8) as the system for the pair $\left(a^{h}, \phi^{h}\right)$ :

$$
\left\{\begin{array}{l}
\partial_{\tau} a^{h}+\nabla \phi^{h} \cdot \nabla a^{h}+\frac{1}{2} a^{h} \Delta \phi^{h}=i \frac{h}{2} \Delta a^{h}, \\
\partial_{\tau} \phi^{h}+\frac{1}{2}\left|\nabla \phi^{h}\right|^{2}+\lambda \tau^{\gamma-2}\left(|y|^{-\gamma} *\left|a^{h}\right|^{2}\right)=0, \\
a_{\mid \tau=h}^{h} \frac{\alpha}{\gamma-\alpha}=a_{0}, \quad \phi_{\mid \tau=h}^{h} \frac{\alpha}{\gamma-\alpha}=0 .
\end{array}\right.
$$

If we approximate the solution of (1.11) up to $h^{1}$ order, that is, if we establish an asymptotics such as

$$
a^{h}=b_{0}+h b_{1}+o\left(h^{1}\right), \quad \quad \phi^{h}=\phi_{0}+h \phi_{1}+o\left(h^{1}\right),
$$

then, by means of (1.10), we immediately obtain the WKB-type approximate solution of $\psi^{h}$. This method is first employed for nonlinear Schrödinger equation with a certain 
class of defocussing local nonlinearity including the cubic nonlinearity $N(y)=|y|^{2} y$ for analytic data [15] and for Sobolev data [18] (see, also $[2,11]$ ), and is extended to the Gross-Pitaevskii equation [3] (see, also [22]) and to the equation with (de)focusing nonlocal nonlinearities: Schrödinger-Poisson equation [1, 23] (see, also [21, 30]) and Hartree equation [9].

Our goal is to justify (1.12). The natural choice of the main part $\left(b_{0}, \phi_{0}\right)$ may be $\left(a^{h}, \phi^{h}\right)_{\mid h=0}$. Letting $h=0$ in (1.11), we obtain a hydrodynamical system

$$
\left\{\begin{array}{l}
\partial_{\tau} b_{0}+\nabla \phi_{0} \cdot \nabla b_{0}+\frac{1}{2} b_{0} \Delta \phi_{0}=0, \\
\partial_{\tau} \phi_{0}+\frac{1}{2}\left|\nabla \phi_{0}\right|^{2}+\lambda \tau^{\gamma-2}\left(|y|^{-\gamma} *\left|b_{0}\right|^{2}\right)=0, \\
b_{0 \mid \tau=0}=a_{0}, \quad \phi_{0 \mid \tau=0}=0 .
\end{array}\right.
$$

For a Sobolev data $a_{0},(1.13)$ has a unique local solution (Theorem 1.3). Then, the main task is to determine $h^{1}$-term $\left(b_{1}, \phi_{1}\right)$. The difficulty of finding $h^{1}$-term lies in the following two respects:

1. The equaiton (1.11) itself depends on $h$ through the term $i \frac{h}{2} \Delta a^{h}$.

2. The initial time of (1.11) tends to $\tau=0$ at a speed $h^{\frac{\alpha}{\gamma-\alpha}}$.

We will see that the first becomes crucial when we consider the asymptotic behavior of the solution on the final layer, and that a suitable choice of $h^{1}$-term is the key for overcoming this difficulty. We use $\left(b_{\text {equ }}, \phi_{\text {equ }}\right)$ defined by (1.18), below, as an $h^{1}$-term and show an asymptotic behavior of $\psi^{h}$ for $\tau \in\left[h^{\frac{\alpha}{\gamma-\alpha}}, T\right]$ when $\alpha \geqslant 1$, where $T$ is independent of $h$ (Theorem 1.3). By (1.9), $\tau \in\left[h^{\frac{\alpha}{\gamma-\alpha}}, T\right]$ is equivalent to $t \in\left[0,1-T^{-1} \varepsilon^{\alpha / \gamma}\right]$, from the initial time to the final layer. On the other hand, the second becomes crucial in the supercritical case $\alpha<1$. This is because the moving speed of initial time becomes too slow. In this case, we need three more kinds of correction terms whose order are between $h^{0}$ and $h^{1}$. With them, we describe the asymptotic behavior also for $\alpha \in] 0,1[$. A heuristic observation on these correction terms is in Section 2.2. The rigorous result is in Theorem 5.1.

The well-prepared data (1.6) is closely related to the second problem listed above. The function $\left(b_{0}, \phi_{0}\right)$ in (1.6) is the solution of (1.13). If we employ the well-prepared data and consider (1.3) with (1.6), then (1.11) changes into

$$
\left\{\begin{array}{l}
\partial_{\tau} a^{h}+\nabla \phi^{h} \cdot \nabla a^{h}+\frac{1}{2} a^{h} \Delta \phi^{h}=i \frac{h}{2} \Delta a^{h}, \\
\partial_{\tau} \phi^{h}+\frac{1}{2}\left|\nabla \phi^{h}\right|^{2}+\lambda \tau^{\gamma-2}\left(|y|^{-\gamma} *\left|a^{h}\right|^{2}\right)=0, \\
a_{\mid \tau=h}^{h} \frac{\alpha}{\gamma-\alpha}=b_{0}\left(h^{\frac{\alpha}{\gamma-\alpha}}\right), \quad \phi_{\mid \tau=h}^{h} \frac{\alpha}{\gamma-\alpha}=\phi_{0}\left(h^{\frac{\alpha}{\gamma-\alpha}}\right) .
\end{array}\right.
$$

The initial time is still moving, however, the only difference between (1.14) and (1.13) is the existence of $i \frac{h}{2} \Delta a^{h}$. Thus, we will see that we do not meet with the second problem any longer. This point is discussed in Sections 5.2.

We also consider the problem of global existence of the solution to (1.13). With the notation $(\rho, v):=\left(\left|b_{0}\right|^{2}, \nabla \phi_{0}\right)$, (1.13) is the compressible Euler equation with time-dependent pressure term of Hartree type:

$$
\left\{\begin{array}{l}
\partial_{\tau} \rho+\operatorname{div}(\rho v)=0, \\
\partial_{\tau} v+(v \cdot \nabla) v+\lambda \tau^{\gamma-2} \nabla\left(|x|^{-\gamma} * \rho\right)=0, \\
\rho_{\mid \tau=0}=\left|a_{0}\right|^{2}, \quad v_{\mid \tau=0}=0 .
\end{array}\right.
$$


We adapt results in $[12,25]$ (see also $[24,28]$ ) to prove that $C^{1}$-solution to (1.15) cannot be global in time, in several situations (Theorems 1.5 and 1.6). This implies (1.12) breaks down before caustic $t=1$ (see Section 2.1).

1.1. Main result I. To state our result precisely, we introduce some notation. For $n \geqslant 3, s>n / 2+1, p \in[1, \infty]$, and $q \in[1, \infty]$, we define a function space $Y_{p, q}^{s}\left(\mathbb{R}^{n}\right)$ by

$$
Y_{p, q}^{s}\left(\mathbb{R}^{n}\right)=\overline{C_{0}^{\infty}\left(\mathbb{R}^{n}\right)}\|\cdot\|_{Y_{p, q}^{s}\left(\mathbb{R}^{n}\right)}
$$

with norm

$$
\|\cdot\|_{Y_{p, q}^{s}\left(\mathbb{R}^{n}\right)}:=\|\cdot\|_{L^{p}\left(\mathbb{R}^{n}\right)}+\|\nabla \cdot\|_{L^{q}\left(\mathbb{R}^{n}\right)}+\left\|\nabla^{2} \cdot\right\|_{H^{s-2}\left(\mathbb{R}^{n}\right)} .
$$

We denote $Y_{p, q}^{s}=Y_{p, q}^{s}\left(\mathbb{R}^{n}\right)$, for short. For $q<n$, we use the notation $q^{*}=n q /(n-q)$. This space $Y_{p, q}^{s}$ is a modification of the Zhidkov space $X^{s}$, which is defined, for $s>n / 2$, by $X^{s}\left(\mathbb{R}^{n}\right):=\left\{f \in L^{\infty}\left(\mathbb{R}^{n}\right) \mid \nabla f \in H^{s-1}\left(\mathbb{R}^{n}\right)\right\}$. The Zhidkov space was introduced in [31] (see, also [13]). Roughly speaking, the exponents $p$ and $q$ in $Y_{p, q}^{s}$ indicate the decay rates at the spatial infinity of a function and of its first derivative, respectively. Moreover, the Zhidkov space $X^{s}$ corresponds to $Y_{\infty, 2}^{s}$ in a sense, if $n \geqslant 3$. We discuss these points more precisely in Section 3.1. We also note that $Y_{2,2}^{s}$ is the usual Sobolev space $H^{s}$. We use the following notation: $Y_{p, q}^{\infty}:=\cap_{s>0} Y_{p, q}^{s}$; for intervals $I_{1}$ and $I_{2}$ of $[1, \infty], Y_{I_{1}, q}^{s}:=\cap_{p \in I_{1}} Y_{p, q}^{s}$ and $Y_{p, I_{2}}^{s}:=\cap_{q \in I_{2}} Y_{p, q}^{s}$. These notation are sometimes used simultaneously, for example $Y_{I_{1}, I_{2}}^{\infty}:=\cap_{s>0, p \in I_{1}, q \in I_{2}} Y_{p, q}^{s}$. We also use the operator

$$
\left|J^{\varepsilon}\right|^{s}=e^{i \frac{|x|^{2}}{2 \varepsilon(t-1)}}|(1-t) \nabla|^{s} e^{-i \frac{|x|^{2}}{2 \varepsilon(t-1)}}
$$

This is the scaled version of the Galilean operator and is suitable for a study of rapid phases other than $e^{i|x|^{2} / 2 \varepsilon(t-1)}$.

We also introduce the following systems for a pair $\left(b_{\text {equ }}, \phi_{\text {equ }}\right)$ :

$$
\left(\begin{array}{l}
\partial_{\tau} b_{\text {equ }}+\nabla \phi_{\text {equ }} \cdot \nabla b_{0}+\nabla \phi_{0} \cdot \nabla b_{\text {equ }}+\frac{1}{2} b_{\text {equ }} \Delta \phi_{0}+\frac{1}{2} b_{0} \Delta \phi_{\text {equ }}=\frac{i}{2} \Delta b_{0} \\
\partial_{\tau} \phi_{\text {equ }}+\nabla \phi_{0} \cdot \nabla \phi_{\text {equ }}+\lambda \tau^{\gamma-2}\left(|y|^{-\gamma} * 2 \operatorname{Re} \overline{b_{0}} b_{\text {equ }}\right)=0
\end{array}\right.
$$

where $\left(b_{0}, \phi_{0}\right)$ is a solution of (1.13). This will be posed with the zero data

$$
b_{\text {equ } \mid \tau=0}=0, \quad \phi_{\text {equ } \mid \tau=0}=0
$$

or the data

$$
b_{\text {equ } \mid \tau=0}=0, \quad \quad \phi_{\text {equ } \mid \tau=0}=\lambda\left(|y|^{-\gamma} *\left|a_{0}\right|^{2}\right) /(\gamma-1) .
$$

Notation 1.1. Let $T>0$ and $X$ be a Banach space. Let $\left\{k_{j}\right\}$ be an increasing sequence of real number, $\phi(t, x) \in C([0, T] ; X)$ be a function, and $\left\{\phi_{j}\right\}$ be a sequence of function in $X$. We write

$$
\phi(t, x) \asymp \sum_{j=1}^{\infty} t^{k_{j}} \phi_{j} \quad \text { in } X
$$


if it holds that

$$
\left\|\phi(t, x)-\sum_{j=1}^{J} t^{k_{j}} \phi_{j}\right\|_{X}=o\left(t^{k_{J}}\right)
$$

as $t \rightarrow 0$ for all $J \geqslant 1$.

We now state our main result. To avoid complicity, here we state the result for $\alpha \geqslant 1$; the linear and nonlinear WKB cases. For the supercritical WKB case $\alpha<1$, see Theorem 5.1.

Assumption 1.2. Let $n \geq 4$ and $\lambda \in \mathbb{R}$. The constants $\gamma$ and $\alpha$ satisfy $\max (1, n / 2-2)<\gamma \leq n-2$ and $0<\alpha<\gamma$, respectively. The initial data $a_{0} \in H^{\infty}$.

Theorem 1.3. Let assumption 1.2 be satisfied. Assume $\alpha \geqslant 1$. Then, there exists an existence time $T>0$ independent of $\varepsilon$. There also exist $\left(b_{0}, \phi_{0}\right),\left(b_{\text {equ }}, \phi_{\text {equ }}\right) \in$ $C\left([0, T] ; H^{\infty} \times Y_{(n / \gamma, \infty],(n /(\gamma+1), \infty]}^{\infty}\right)$ such that:

1. $\phi_{0}(\tau, y) \asymp \sum_{j=1}^{\infty} \tau^{\gamma j-1} \varphi_{j}(y)$ in $Y_{(n / \gamma, \infty],(n /(\gamma+1), \infty]}^{\infty}$.

2. The solution $u^{\varepsilon}$ to (1.3) with (1.4) satisfies the following asymptotics for all $s \geqslant 0$ :

$$
\sup _{t \in\left[0,1-T^{-1} \varepsilon^{\alpha / \gamma}\right]}\left\|\left|J^{\varepsilon}\right|^{S}\left(u^{\varepsilon}(t) e^{-i \Phi^{\varepsilon}(t)}-\frac{1}{(1-t)^{n / 2}} A^{\varepsilon}(t) e^{i \frac{|\cdot|^{2}}{2 \varepsilon(t-1)}}\right)\right\|_{L^{2}} \rightarrow 0
$$

as $\varepsilon \rightarrow 0$ with

$$
\Phi^{\varepsilon}(t, x)=\varepsilon^{\frac{\alpha}{\gamma}-1} \phi_{0}\left(\frac{\varepsilon^{\frac{\alpha}{\gamma}}}{1-t}, \frac{x}{1-t}\right)
$$

and

$$
A^{\varepsilon}(t, x)=b_{0}\left(\frac{\varepsilon^{\frac{\alpha}{\gamma}}}{1-t}, \frac{x}{1-t}\right) \exp \left(i \phi_{\text {equ }}\left(\frac{\varepsilon^{\frac{\alpha}{\gamma}}}{1-t}, \frac{x}{1-t}\right)\right),
$$

where $\left(b_{0}, w_{0}\right)$ solves (1.13) and $\left(b_{\text {equ }}, \phi_{\text {equ }}\right)$ solves (1.18) with (1.19) if $\alpha>1$ and with (1.20) if $\alpha=1$.

REMARK 1.4.

1. By the definition of “ $\asymp$ " sign, the expansion $\phi_{0}(\tau, y) \asymp \sum_{j=1}^{\infty} \tau^{\gamma j-1} \varphi_{j}(y)$ implies

$$
\phi_{0}(\tau)=\sum_{j=1}^{J} \tau^{\gamma j-1} \varphi_{j}+o\left(\tau^{\gamma J-1}\right) \quad \text { in } Y_{(n / \gamma, \infty],(n /(\gamma+1), \infty]}^{\infty}
$$

as $\tau \rightarrow 0$ for all $J \geqslant 1$.

2. In Theorem 1.3, we only need $a_{0} \in H^{s_{0}}$ with some $s_{0}>n / 2+3$. Then, $\left(b_{0}, \phi_{0}\right)$ belongs to $C\left([0, T], H^{s_{0}} \times Y_{(n / \gamma, \infty],(n /(\gamma+1), \infty]}^{s_{0}+2}\right)$ and $\left(b_{\text {equ }}, \phi_{\text {equ }}\right)$ belongs to $C\left([0, T], H^{s_{0}-2} \times Y_{(n / \gamma, \infty],(n /(\gamma+1), \infty]}^{s_{0}}\right)$. The asymptotics (1.21) holds for any $s \in\left[0, s_{0}-4\right]$. 
3. All $\varphi_{j}$ are given explicitly (but inductively) in terms of $a_{0}$. For example, $\varphi_{1}=\lambda\left(|x|^{-\gamma} *\left|a_{0}\right|^{2}\right) /(1-\gamma)$ and

$$
\begin{aligned}
\varphi_{2}= & -\frac{\lambda^{2}}{2(\gamma-1)^{2}(2 \gamma-1)}\left|\nabla\left(|x|^{-\gamma} *\left|a_{0}\right|^{2}\right)\right|^{2} \\
& -\frac{\lambda^{2}}{\gamma(\gamma-1)(2 \gamma-1)}\left(|x|^{-\gamma} *\left(\nabla \cdot\left(\left|a_{0}\right|^{2} \nabla\left(|x|^{-\gamma} *\left|a_{0}\right|^{2}\right)\right)\right)\right.
\end{aligned}
$$

(see, Proposition 4.2).

4. Even if the system (1.18) is posed with zero initial condition, its solution $\left(b_{\text {equ }}, \phi_{\text {equ }}\right)$ is not identically zero because of the presence of the (nontrivial) external force $\frac{i}{2} \Delta b_{0}$.

5. The choice of the initial data (1.20) is the key for the analysis in the case $\alpha=1$. We discuss this point more precisely in Section 2.2.

The asymptotics (1.21) reads

$$
u^{\varepsilon}(t, x) \sim \frac{1}{(1-t)^{n / 2}} A^{\varepsilon}(t, x) e^{i \Phi^{\varepsilon}(t, x)} e^{i \frac{|x|^{2}}{2 \varepsilon(t-1)}}
$$

as $\varepsilon \rightarrow 0$. Indeed, this holds in $L^{\infty}\left(\left[0,1-T^{-1} \varepsilon^{\alpha / \gamma}\right] ; L^{2}\right)$. This explains the cascade of phase shifts. We consider the case $1<\alpha(<\gamma)$. Combining (1.24) and (1.22), we have

$$
\Phi^{\varepsilon}(t, x)=\sum_{j=1}^{J} \frac{\varepsilon^{\alpha j-1}}{(1-t)^{\gamma j-1}} \varphi_{j}\left(\frac{x}{1-t}\right)+o\left(\frac{\varepsilon^{\alpha J-1}}{(1-t)^{\gamma J-1}}\right) .
$$

We set $g_{j}^{\varepsilon}(t, x)=\frac{\varepsilon^{j \alpha-1}}{(1-t)^{j \gamma-1}} \varphi_{j}\left(\frac{x}{1-t}\right)$. Then, the above asymptotics yields

$$
\begin{array}{rlrl}
\Phi^{\varepsilon}(t, x) & \sim 0 & & \text { for } 1-t \gg \varepsilon^{\frac{\alpha-1}{\gamma-1}}, \\
\Phi^{\varepsilon}(t, x) & \sim g_{1}^{\varepsilon}(t, x) & & \text { for } 1-t \gg \varepsilon^{\frac{2 \alpha-1}{2 \gamma-1}}, \\
\Phi^{\varepsilon}(t, x) & \sim g_{1}^{\varepsilon}(t, x)+g_{2}^{\varepsilon}(t, x) & & \text { for } 1-t \gg \varepsilon^{\frac{3 \alpha-1}{3 \gamma-1}} \\
\vdots & & \vdots \\
\Phi^{\varepsilon}(t, x) & \sim \sum_{j=1}^{J} g_{j}^{\varepsilon}(t, x) & & \text { for } 1-t \gg \varepsilon^{\frac{J \alpha-1}{J \gamma-1}}
\end{array}
$$

as $\varepsilon \rightarrow 0$. On the other hand, the amplitude $A^{\varepsilon}$ satisfies

$$
A^{\varepsilon}(t, x)=a_{0}\left(\frac{x}{1-t}\right)+o(1)
$$


as $\varepsilon^{\frac{\alpha}{\gamma}} /(1-t) \rightarrow 0$. Substitute these expansions to (1.25) to obtain

$$
\begin{aligned}
u^{\varepsilon} & \sim v_{\operatorname{lin}}^{\varepsilon}=\frac{1}{(1-t)^{n / 2}} a_{0}\left(\frac{x}{1-t}\right) e^{i \frac{|x|^{2}}{2 \varepsilon(t-1)}} & & \text { for } 1-t \gg \varepsilon^{\frac{\alpha-1}{\gamma-1}}, \\
u^{\varepsilon} & \sim v_{\operatorname{lin}}^{\varepsilon} e^{i g_{1}^{\varepsilon}(t, x)} & & \text { for } 1-t \gg \varepsilon^{\frac{2 \alpha-1}{2 \gamma-1}}, \\
u^{\varepsilon} & \sim v_{\operatorname{lin}}^{\varepsilon} e^{i g_{1}^{\varepsilon}(t, x)+i g_{2}^{\varepsilon}(t, x)} & & \text { for } 1-t \gg \varepsilon^{\frac{3 \alpha-1}{3 \gamma-1}}, \\
& \vdots & & \vdots \\
u^{\varepsilon} & \sim v_{\operatorname{lin}}^{\varepsilon} e^{i \sum_{j=1}^{J} g_{j}^{\varepsilon}(t, x)} & & \text { for } 1-t \gg \varepsilon^{\frac{J \alpha-1}{J \gamma-1}},
\end{aligned}
$$

Recall that $v_{\text {lin }}^{\varepsilon}$, given in (1.5), is the approximate solution for the linear solution $w^{\varepsilon}$. One sees that the solution behaves like a free solution in the region $1-t \gg \varepsilon^{\frac{\alpha-1}{\gamma-1}}$ where the initial time $t=0$ lies, and that, at each boundary layer of size $1-t \sim \varepsilon^{\frac{J \alpha-1}{J \gamma-1}}$ (the $J$-th boundary layer), a new phase associated with $g_{J}^{\varepsilon}$ becomes relevant.

1.2. Main result II. Our next result is the non-existence of a global solution to (1.13). We further assume the radial symmetry and $\gamma=n-2(n \geqslant 3)$ in (1.15). A suitable change of $\lambda$ yields the radial compressible Euler-Poisson equations

$$
\left\{\begin{array}{l}
\partial_{\tau}\left(\rho r^{n-1}\right)+\partial_{r}\left(\rho v r^{n-1}\right)=0, \\
\partial_{\tau} v+v \partial_{r} v-\lambda \tau^{n-4} \partial_{r} V_{\mathrm{p}}=0, \\
\partial_{r}\left(r^{n-1} \partial_{r} V_{\mathrm{p}}\right)=\rho r^{n-1}, \\
\rho_{\mid \tau=0}=\left|a_{0}\right|^{2}, \quad v_{\mid \tau=0}=0,
\end{array}\right.
$$

where $r:=|x|$. We define the "mean mass" $M_{0}$ in $\{|x| \leqslant r\}$ by

$$
M_{0}(r):=\frac{1}{r^{n}} \int_{0}^{r}\left|a_{0}(s)\right|^{2} s^{n-1} d s .
$$

THEOREM 1.5. Let $\lambda<0$ and $n \geqslant 4$. For every nonzero initial amplitude $a_{0} \in C^{1}$, the solution to (1.26) breaks down no latter than

$$
T^{*}=\left(\frac{(n-2)(n-3)}{|\lambda| \sup _{r \geqslant 0} M_{0}(r)}\right)^{\frac{1}{n-2}}<\infty .
$$

Theorem 1.6 ([12], Theorem 5.10). Let $\lambda>0$ and $n=4$. The radial $C^{1}$-solution $(a, v)$ to $(1.26)$ is global if and only if the initial amplitude $a_{0} \in C^{1}$ satisfies

$$
\left|a_{0}(r)\right|^{2} \geqslant 2 M_{0}(r)=\frac{2}{r^{4}} \int_{0}^{r}\left|a_{0}(s)\right|^{2} s^{3} d s
$$

for all $r \geqslant 0$. In particular, if $a_{0} \in L^{2}\left(\mathbb{R}^{4}\right)$ then the solution breaks down in finite time. Moreover, the critical time is given by

$$
\tau_{c}=\left(\frac{2}{\lambda \max _{r>0}\left(2 M_{0}(r)-\left|a_{0}(r)\right|^{2}\right)}\right)^{\frac{1}{2}} .
$$


EXAMPLE 1.7 (An example of finite-time breakdown). Consider the equation (1.26). Let $n=4$ and $\lambda>0$. Suppose

$$
a_{0}(x)=a_{0}(r)=r^{-\frac{5}{2}} e^{-\frac{1}{2 r}} .
$$

Then, an elementary calculation shows

$$
M_{0}(r)=r^{-4} e^{-\frac{1}{r}}
$$

and so that $2 M_{0}(r)-\left|a_{0}\right|^{2}=(2 r-1) r^{-5} e^{-\frac{1}{r}}$ takes its maximum at $r_{0}=(7+\sqrt{17}) / 16=$ $0.6951 \ldots$ which is the root of

$$
8 r_{0}^{2}-7 r_{0}+1=0
$$

By Theorem 1.6, the critical time is

$$
\tau_{c}=\left(\frac{2}{|\lambda|\left(2 M_{0}\left(r_{0}\right)-\left|a_{0}\left(r_{0}\right)\right|^{2}\right)}\right)^{\frac{1}{2}}=\sqrt{\frac{3405+827 \sqrt{17}}{\lambda 2^{13}}} e^{\frac{3(7-\sqrt{17})}{32}} .
$$

In fact, the solution to (1.26) is given by

$$
\begin{aligned}
|a|^{2}(\tau, X(t, R)) & =\frac{2 R^{2}}{X^{2}(\tau, R)\left(2 R^{5} e^{\frac{1}{R}}-\lambda(2 R-1) \tau^{2}\right)}, \\
v(\tau, X(\tau, R)) & =\frac{\lambda \tau}{X(\tau, R) R^{2} e^{\frac{1}{R}}},
\end{aligned}
$$

where

$$
X(\tau, R)=R \sqrt{1+\lambda R^{-4} e^{-\frac{1}{R}} \tau^{2}} .
$$

At the time $\tau=\tau_{c}$, the characteristic curves "touch" at $r=r_{c}:=X\left(t_{c}, r_{0}\right)$, that is, we have $\left(\partial_{R} X\right)\left(\tau_{c}, r_{0}\right)=0$, which is one of the sufficient and necessary condition for finite-time breakdown (see, [12]). More explicitly, we can see that, as $\tau$ tends to $\tau_{c}$, the amplitude $|a|^{2}$ blows up at $r_{c}$ since the denominator

$$
2 r_{0}^{5} e^{\frac{1}{r_{0}}}-\lambda\left(2 r_{0}-1\right) t^{2}
$$

tends to zero as $\tau \rightarrow \tau_{c}$. We illustrate the calculation in Remark 6.2.

In this example, $a_{0} \in H^{\infty} \subset C^{1}$ and so Theorem 1.3 holds. We see that, however, the asymptotics (1.21) is valid only for $\varepsilon^{\frac{\alpha}{\gamma}} /(1-t)<\tau_{c}$ and it cannot hold for $\varepsilon^{\frac{\alpha}{\gamma}} /(1-$ $t) \geqslant \tau_{c}$.

The rest of the paper is organized as follows. In Section 2, we make a summary of the results in this paper with previous results. Section 3 is devoted to preliminary results. We prove Theorem 1.3 in Section 4. The strategy of the proof is illustrated rather precisely in Section 4.1. In Section 5, we treat the supercritical WKB case $\alpha<1$ (Theorem 5.1). The well-prepared data is discussed in Section 5.2 Finally, we prove Theorems 1.5 and 1.6 in Section 6. 


\section{Summary.}

2.1. Cascade of phase shifts in the linear WKB case. We first discuss about the linear WKB case $\alpha>1$. According to Theorems 1.3, 1.5, and 1.6, we summarize the result in this case as follows. Recall several boundary layers:

$$
\begin{aligned}
\text { initial time: } & t=0 & \longleftrightarrow 1-t=1, \\
\text { first layer: } & t=1-\Lambda_{1} \varepsilon^{\frac{\alpha-1}{\gamma-1}} & \longleftrightarrow 1-t=\Lambda_{1} \varepsilon^{\frac{\alpha-1}{\gamma-1}}, \\
J \text {-th layer: } & t=1-\Lambda_{J} \varepsilon^{\frac{J \alpha-1}{J \gamma-1}} & \longleftrightarrow 1-t=\Lambda_{J} \varepsilon^{\frac{J \alpha-1}{J \gamma-1}} \\
\text { final layer: } & t=1-T^{-1} \varepsilon^{\frac{\alpha}{\gamma}} & \longleftrightarrow 1-t=T^{-1} \varepsilon^{\frac{\alpha}{\gamma}}
\end{aligned}
$$

where $\Lambda_{J}$ and $T$ are positive constants.

- From the initial time to the first layer. Before the first layer, that is, for $1-t \gg \varepsilon^{\frac{\alpha-1}{\gamma-1}}$, the behavior of the solution is the same as in the linear case at leading order. Indeed, the asymptotics (1.21) implies that the solution behaves like $v_{\operatorname{lin}}^{\varepsilon}$ defined in (1.5) for $1-t \gg \varepsilon^{\frac{\alpha-1}{\gamma-1}}$. The phase shifts disappear: From (1.22) and the time expansion $\phi_{0}(\tau) \asymp \sum_{j=1}^{\infty} \tau^{\gamma j-1} \varphi_{j}$, we see

$$
\left\|\Phi^{\varepsilon}(t)\right\|_{L^{\infty}\left(\mathbb{R}^{n}\right)} \leqslant C \varepsilon^{\frac{\alpha}{\gamma}-1} \sum_{j=1}^{\infty}\left(\frac{\varepsilon^{\frac{\alpha}{\gamma}}}{1-t}\right)^{\gamma j-1}\left\|\varphi_{j}\right\|_{L^{\infty}\left(\mathbb{R}^{n}\right)} \ll 1
$$

if $1-t \gg \varepsilon^{\frac{\alpha-1}{\gamma-1}}$. Moreover, the amplitude tends to a rescaling of $a_{0}$ : By (1.23),

$$
A^{\varepsilon}(t, x) \rightarrow b_{0}\left(0, \frac{x}{1-t}\right) \exp \left(i \phi_{\text {equ }}\left(0, \frac{x}{1-t}\right)\right)=a_{0}\left(\frac{x}{1-t}\right)
$$

since $\varepsilon^{\alpha / \gamma} /(1-t) \ll 1$ for $1-t \gg \varepsilon^{\frac{\alpha-1}{\gamma-1}}$. This agrees with the analysis in [26]. On the other hand, on the first layer $1-t=\Lambda_{1} \varepsilon^{\frac{\alpha-1}{\gamma-1}}$ the nonlinear effect becomes relevant. The term $\tau^{\gamma-1} \varphi_{1}$ in the $\operatorname{sum}^{1} \sum_{j=1}^{\infty} \tau^{\gamma j-1} \varphi_{j}$ is no longer negligible.

- From the first layer to the $J$-th layer. Soon after the first layer, the solution becomes strongly oscillatory by $\tau^{\gamma-1} \varphi_{1}$. Between the first and the second layers, only $\tau^{\gamma-1} \varphi_{1}$ is effective because

$$
\begin{aligned}
\| \Phi^{\varepsilon}(t)-\varepsilon^{\frac{\alpha}{\gamma}-1}\left(\frac{\varepsilon^{\frac{\alpha}{\gamma}}}{1-t}\right)^{\gamma-1} \varphi_{1}\left(\frac{\cdot}{1-t}\right) & \|_{L^{\infty}\left(\mathbb{R}^{n}\right)} \\
& \leqslant \varepsilon^{\frac{\alpha}{\gamma}-1} \sum_{j=2}^{\infty}\left(\frac{\varepsilon^{\frac{\alpha}{\gamma}}}{1-t}\right)^{\gamma j-1}\left\|\varphi_{j}\right\|_{L^{\infty}\left(\mathbb{R}^{n}\right)} \ll 1
\end{aligned}
$$

holds if $1-t \gg \varepsilon^{\frac{2 \alpha-1}{2 \gamma-1}}$. When we reached to the second layer $1-t=\Lambda_{2} \varepsilon^{\frac{2 \alpha-1}{2 \gamma-1}}$, the phase $\tau^{2 \gamma-1} \varphi_{2}$ become relevant. Similarly, at each $J$-th layer $1-t=\Lambda_{J} \varepsilon^{\frac{J \alpha-1}{J \gamma-1}}$ new phase $\varphi_{J}$ becomes relevant. This is the cascade of phase shift. In this regime, $\varepsilon^{\frac{\alpha}{\gamma}} /(1-t)$ converges to zero as $\varepsilon \rightarrow 0$, and so that the amplitude still stays the linear one.

\footnotetext{
${ }^{1}$ This sum is the formal one: Here, we say "sum" in the sense that $\phi_{0}(\tau) \asymp \sum_{j=1}^{\infty} \tau^{\gamma j-1} \varphi_{j}$.
} 
- On the final layer. After a countable number of boundary layers, we reach to the final layer. In this layer, $\varepsilon^{\frac{\alpha}{\gamma}} /(1-t)$ does not tend to zero any longer. Therefore, the asymptotics of amplitude changes into a nonlinear one. It turns out that the ratio $T:=\varepsilon^{\alpha / \gamma} /(1-t)$ plays the crucial role. If $T \geqslant 0$ is small, then the asymptotic behavior of the solution is described by the asymptotics (1.21). It is essential to use $\Phi^{\varepsilon}$ and $A^{\varepsilon}$ defined in (1.22) and (1.23), respectively. Thanks to the nontrivial remainder term given by $\phi_{\text {equ }},(1.21)$ gives the asymptotic behavior of the solution on the final layer.

On the other hand, (1.21) breaks down at $T=T^{*}<\infty$ in several cases (Theorems 1.5 and 1.6). It is because the nonlinear effects cause a formation of singularity. These focal points are moving: If the focal point is

$$
\left(\frac{\varepsilon^{\frac{\alpha}{\gamma}}}{1-t_{c}}, \frac{x_{c}}{1-t_{c}}\right)=\left(T^{*}, X^{*}\right)
$$

then, in the $(t, x)$-coordinates, this focal point is

$$
t_{c}=1-\left(T^{*}\right)^{-1} \varepsilon^{\frac{\alpha}{\gamma}}, \quad x_{c}=X^{*}\left(T^{*}\right)^{-1} \varepsilon^{\frac{\alpha}{\gamma}},
$$

which tends to $(t, x)=(1,0)$ as $\varepsilon \rightarrow 0$. Example 1.7 gives an example of this type blowup.

- After the final layer. It remains open what happens after the breakdown of (1.21). However, we can at least expect that more rapid oscillations might not appear after the final layer. This is because, in the case of $\lambda>0,2 \leqslant \gamma<4$, and $\alpha \in] \max (\gamma / 2,1), \gamma\left[\right.$, the order of the upper bound of $\left\|J^{\varepsilon} u^{\varepsilon}\right\|_{L^{2}}$ stays $\varepsilon^{1-\alpha / \gamma}$ even after the caustic ([26]). Recall that $J^{\varepsilon}=e^{i|x|^{2} / 2 \varepsilon(t-1)} i(t-1) \nabla e^{-i|x|^{2} / 2 \varepsilon(t-1)}$ filters out the main quadratic phase. Therefore, this divergent upper bound implies that the order of magnitude of energy of the oscillation other than the main quadratic phase is at most $\varepsilon^{1-\alpha / \gamma}$.

2.2. Cascade of phase shifts in the nonlinear and supercritical WKB case. We now turn to the case $\alpha<1$, the supercritical WKB case. Our goal is to obtain a WKB-type approximation similar to (1.21), which explains the cascade of phase shifts and gives the asymptotic profile of the solution before and on the final layer. We do not use modified the initial data (1.6) and keep working with (1.3)-(1.4). As stated above, the analysis of (1.3)-(1.4) is reduced to the analysis of (1.11) via the transform (1.7). Recall that $h=\varepsilon^{1-\alpha / \gamma}$. The difficulty is the following:

1. The equaiton (1.11) itself depends on $h$ through the term $i \frac{h}{2} \Delta a^{h}$.

2. The initial time of (1.11) tends to $\tau=0$ at a speed $h^{\frac{\alpha}{\gamma-\alpha}}$.

The second is the main point of the supercritical case $\alpha<1$. In this section, we discuss by heuristic arguments what happens, what is the problem, and how we can overcome it. Then, it turns out that the situation becomes more complicated, and the cascade of phase shifts phenomena involves more phase shifts and boundary layers than the linear WKB case. The rigorous result is in Theorem 5.1 (see, also Remark $5.3)$.

- The transpose of the initial time and the first boundary layer. We see from (2.1) that the relation $\alpha<1$ causes the transpose of the initial time $t=0$ and the first boundary layer, that is, the initial time $t=0$ lies beyond the first boundary layer $t=1-\Lambda_{1} \varepsilon^{\frac{\alpha-1}{\gamma-1}}$ because $\Lambda_{1} \varepsilon^{\frac{\alpha-1}{\gamma-1}} \gg 1$ for small $\varepsilon$. It means that there is no linear regime and the behavior of the solution involves nonlinear effects at leading order soon after the initial time. 
- The nonlinear behavior of the solution at the initial time. As a matter of fact, the behavior of $u^{\varepsilon}$ is already "nonlinear" at $t=0$. More precisely, the principal part of the phase shift of the solution $u^{\varepsilon}$ other than $\exp \left(i|x|^{2} / 2 \varepsilon(t-1)\right.$ ) (we call this as a principal nonlinear phase of $u^{\varepsilon}$ ) for $\alpha<1$ is given by

$$
\exp \left(i \varepsilon^{\frac{\alpha}{\gamma}-1} \phi_{0}\left(\frac{\varepsilon^{\frac{\alpha}{\gamma}}}{1-t}, \frac{x}{1-t}\right)\right)
$$

where $\phi_{0}$ is the solution of (1.13). Here we remark that the principal nonlinear phase is the same as in the case $\alpha>1$ (In Theorem 1.3, we denote this by $\exp \left(i \Phi^{\varepsilon}\right)$ ). Since $\phi_{0}$ is given as a solution of (1.13), the shape of $\phi_{0}$ is completely independent of $\alpha$. Moreover, the choice of $\phi_{0}$ is natural because the function $\phi_{0}$ is the unique limit of the phase function $\phi^{h}$ which solves (1.11) with $a^{h}$. Recall that $\psi^{h}=a^{h} \exp \left(i \phi^{h} / h\right)$ is an exact solution of (1.8). Using the expansion $\phi_{0}(\tau) \asymp \sum_{j=1}^{\infty} \tau^{\gamma j-1} \varphi_{j}(y)$, we have

$$
\varepsilon^{\frac{\alpha}{\gamma}-1} \phi_{0}\left(\frac{\varepsilon^{\frac{\alpha}{\gamma}}}{1-t}\right) \sim O\left(\varepsilon^{\frac{\alpha}{\gamma}-1}\left(\frac{\varepsilon^{\frac{\alpha}{\gamma}}}{1-t}\right)^{\gamma-1}\right)
$$

as long as $\varepsilon^{\frac{\alpha}{\gamma}} /(1-t) \ll 1$. If the right hand side is small, then the phase shift caused by nonlinear effects is negligible and so the solution behaves like the linear solution. However, the right hand side is $O\left(\varepsilon^{\alpha-1}\right)$ at $t=0$, which is not small if $\alpha<1$. In this sense, the behavior of $u^{\varepsilon}$ is nonlinear at $t=0$.

- The initial condition as a constraint. On the other hand, we always have $u^{\varepsilon}(0, x)=a_{0}(x) \exp \left(-i \frac{|x|^{2}}{2 \varepsilon}\right)$ since, as stated above, we do not modify the initial data and keep working with (1.4). This initial condition seems to be quite natural and the simplest one for this problem. This is true if $\alpha>1$. However, in the supercritical case $\alpha<1$, the meaning of this condition slightly changes and this condition becomes a sort of constraint: The appearing nonlinear effects must disappear at $t=0$. To achieve this constraint, we need to employ some more nontrivial phase shifts as correction terms in order to cancel out the nonlinear effect at $t=0$. This modification with correction terms is the heart of the matter. The main difference between two initial data (1.4) and (1.6) is this point. A use of (1.6) enables us to leave the behavior of the solution at the initial time nonlinear. Hence, we do not need any correction term.

- A formal construction of correction terms. Intuitively, this modification is done as follows: First, we replace $\phi_{0}(\tau, y)$ in $(2.2)$ by $\phi_{0}(\tau, y)-\sum_{j=1}^{k-1} \tau^{\gamma j-1} \varphi_{j}(y)$ for some $k \geqslant 2$. This yields the following modified principal nonlinear phase

$$
\exp \left(i \varepsilon^{\frac{\alpha}{\gamma}-1}\left[\phi_{0}\left(\frac{\varepsilon^{\frac{\alpha}{\gamma}}}{1-t}, \frac{x}{1-t}\right)-\sum_{j=1}^{k-1}\left(\frac{\varepsilon^{\frac{\alpha}{\gamma}}}{1-t}\right)^{\gamma j-1} \varphi_{j}\left(\frac{x}{1-t}\right)\right]\right) .
$$

At $t=0$, it holds that

$$
\varepsilon^{\frac{\alpha}{\gamma}-1}\left[\phi_{0}\left(\varepsilon^{\frac{\alpha}{\gamma}}\right)-\sum_{j=1}^{k-1}\left(\varepsilon^{\frac{\alpha}{\gamma}}\right)^{\gamma j-1} \varphi_{j}\right]=O\left(\varepsilon^{\alpha k-1}\right) .
$$

Therefore, if we take $k$ large enough then the modified approximate solution which the above principal phase (2.3) gives possesses the desired two properties: The leading 
term of the principal nonlinear phase is the same as (2.2); and the phase shifts tend to zero at the initial time $t=0$. Of course, this simple modification is too rude and so valid only in the small neighborhood of $t=0$. Hence, to obtain an approximation also outside the small neighborhood, we replace each $\varphi_{j}(y)$ by a function $-\phi_{\text {pha, } j}(\tau, y)$ which solves a kind of $j$-th linearized system of (1.11) with $-\phi_{\text {pha, } j}(0)=\varphi_{j}$. This yields the principal nonlinear phase like

$$
\exp \left(i \varepsilon^{\frac{\alpha}{\gamma}-1}\left[\phi_{0}\left(\frac{\varepsilon^{\frac{\alpha}{\gamma}}}{1-t}, \frac{x}{1-t}\right)+\sum_{j=1}^{k-1}\left(\varepsilon^{\frac{\alpha}{\gamma}}\right)^{\gamma j-1} \phi_{\text {pha }, j}\left(\frac{t \varepsilon^{\frac{\alpha}{\gamma}}}{1-t}, \frac{x}{1-t}\right)\right]\right)
$$

We note that $t \varepsilon^{\frac{\alpha}{\gamma}} /(1-t)=\varepsilon^{\frac{\alpha}{\gamma}} /(1-t)-\varepsilon^{\frac{\alpha}{\gamma}}$, which is zero at $t=0$.

- Three kinds of correction terms. In fact, the above modified principal nonlinear phase (2.4) is still insufficient. We need two more kinds of correction terms which are essentially different from $\phi_{\mathrm{pha}, j}$. Now, let us list all kinds of correction terms which we use:

1. Correction from phase. The first one is the above $\phi_{\mathrm{pha}, j}$. They satisfy $\phi_{\text {pha }, j}(0)=-\varphi_{j}$ and remove the bad part of $\phi_{0}$.

2. Correction from amplitude. The amplitude $b_{0}$ which pairs $\phi_{0}$ via (1.13) has the expansion $b_{0}(\tau) \asymp a_{0}+\sum_{j=1}^{\infty} \tau^{\gamma j} a_{j}$, where $a_{j}$ is a function of space defined by $a_{0}$ (see, Proposition 4.2). Hence, the principal part of the amplitude of $u^{\varepsilon}$ (principal amplitude of $u^{\varepsilon}$ ) is

$$
b_{0}\left(\frac{\varepsilon^{\frac{\alpha}{\gamma}}}{1-t}, \frac{x}{1-t}\right) \sim a_{0}\left(\frac{x}{1-t}\right)+\sum_{j=1}^{\infty}\left(\frac{\varepsilon^{\frac{\alpha}{\gamma}}}{1-t}\right)^{\gamma j} a_{j}\left(\frac{x}{1-t}\right)
$$

for $\varepsilon^{\frac{\alpha}{\gamma}} /(1-t) \ll 1$. In particular, at $t=0$ we have

$$
b_{0}\left(\varepsilon^{\frac{\alpha}{\gamma}}, x\right) \sim a_{0}(x)+\sum_{j=1}^{k-1} \varepsilon^{\alpha j} a_{j}+O\left(\varepsilon^{\alpha k}\right) .
$$

The principal amplitude converges to the given initial data for all $\alpha>0$, and so one might expect this is harmless. However, it is understood that, when we try to get the pointwise estimate of solution via Grenier's method, we must take $\varepsilon^{1}$-term of the initial amplitude into account because it affects (implicitly) the approximate solution at leading order (see, $[2,9,18])$. Therefore, we must remove $\varepsilon^{\alpha j} a_{j}$ for all $j \geqslant 1$ such that $\alpha j \leqslant 1$ from (2.5), otherwise the approximate solution will differ outside a small neighborhood of $t=0$. To do this, we construct $b_{\mathrm{amp}, j}$ as a solution to a kind of $j$-th linearized system of (1.11) with the condition $b_{\mathrm{amp}, j}(0)=-a_{j}$. At that time, there appear a phase correction $\phi_{\mathrm{amp}, j}$ associated with $b_{\mathrm{amp}, j}$ via the system which $b_{\mathrm{amp}, j}$ solves.

3. Correction from interaction. The third one comes from the structure of (1.11). As stated in introduction, the problem boils down to determining the asymptotic behavior of the solution $\left(a^{h}, \phi^{h}\right)$ to $(1.11)$ up to $O\left(h^{1}\right)$. Suppose that the solution has two terms $\left(h^{p_{1}} b_{1}, h^{p_{1}} \phi_{1}\right)$ and $\left(h^{p_{2}} b_{2}, h^{p_{2}} \phi_{2}\right)$ in its asymptotic expansion as $h \rightarrow 0$. Then, the quadratic terms in (1.11) produce nontrivial $h^{p_{1}+p_{2}}$-terms. For example, $\nabla \phi^{h} \cdot \nabla a^{h}$ has the terms $h^{p_{1}+p_{2}} \nabla \phi_{1} \cdot \nabla b_{2}$ and $h^{p_{1}+p_{2}} \nabla \phi_{2} \cdot \nabla b_{1}$ in its expansion. Again by (1.11), this implies that 
$\left(\partial_{t} b^{h}, \partial_{t} \phi^{h}\right)$ (and so $\left(b^{h}, \phi^{h}\right)$ itself) also contains a $h^{p_{1}+p_{2}}$-term in its expansion. Repeating this argument, we see that $\left(b^{h}, \phi^{h}\right)$ has $h^{p}$-terms for all $p$ given by $p=l p_{1}+m p_{2}$ with integers $l, m \geqslant 0$ such that $l+m \geqslant 1$. So far, we have already obtained two kinds of correction terms, $\phi_{\mathrm{pha}, j}$ and $\phi_{\mathrm{amp}, j}$. Therefore, they interact each other to produce the third correction terms $\phi_{\text {int }, j}$.

- The supercritical cascade of phase shifts. We are now in a position to understand the cascade of phase shifts phenomena in the supercritical case. With the above three kinds of correction terms, we can describe the asymptotic behavior of the solution before the final layer. Recall that, in the linear WKB case $\alpha>1$, the principal nonlinear phase is given by only one phase function $\phi_{0}(\tau, y)$, and the notion of boundary layer comes from its expansion with respect to $\tau$ around $\tau=0$. In this case, not only $\phi_{0}$ but also all $\phi_{\mathrm{pha}, i}, \phi_{\mathrm{amp}, j}, \phi_{\mathrm{int}, k}$ produce a countable number of similar boundary layers. Thus, the cascade of phase shifts involves much "more" phase shifts and boundary layers than the linear WKB case.

- One more correction at the final boundary layer. So far, the second difficulty listed in the beginning of this section is solved by three kinds of correction terms $\phi_{\mathrm{pha}, i}, \phi_{\mathrm{amp}, j}$, and $\phi_{\mathrm{int}, j}$. We can describe with them the asymptotic behavior of the solution before the final layer. To give it also on the final layer, we need one more correction term, $\phi_{\text {equ }}$, as in the case $\alpha>1$. This correction term defeats the first difficulty. This solves (1.18)-(1.19) and so it is independent of $\alpha$. When $\alpha=1$, $\phi_{\text {equ }}$ changes in to a solution of (1.18)-(1.20) (see Theorem 1.3). We next address why we need a modified $\phi_{\text {equ }}$.

- Resonance of correction terms and the nonlinear WKB case. Some of the correction terms associated with $\phi_{\mathrm{pha}, i}, \phi_{\mathrm{amp}, j}, \phi_{\mathrm{int}, k}$, and $\phi_{\text {equ }}$ may have the same order. This phenomena is a kind of resonance. The nonlinear case $\alpha=1$ is the simplest example. In this case, it happens that $\phi_{\text {pha, } 1}$ and $\phi_{\text {equ }}$ have the same order. Recall that if $\alpha=1$ then we work with the modified $\widetilde{\phi}_{\text {equ }}$ solving (1.18)-(1.20),

$$
\left(\begin{array}{l}
\partial_{\tau} \widetilde{b}_{\text {equ }}+\nabla \widetilde{\phi}_{\text {equ }} \cdot \nabla b_{0}+\nabla \phi_{0} \cdot \nabla \widetilde{b}_{\text {equ }}+\frac{1}{2} \widetilde{b}_{\text {equ }} \Delta \phi_{0}+\frac{1}{2} b_{0} \Delta \widetilde{\phi}_{\text {equ }}=\frac{i}{2} \Delta b_{0}, \\
\partial_{\tau} \widetilde{\phi}_{\text {equ }}+\nabla \phi_{0} \cdot \nabla \widetilde{\phi}_{\text {equ }}+\lambda \tau^{\gamma-2}\left(|y|^{-\gamma} * 2 \operatorname{Re} \widetilde{b}_{0} \widetilde{b}_{\text {equ }}\right)=0, \\
\widetilde{b}_{\text {equ } \mid \tau=0} \equiv 0, \quad \widetilde{\phi}_{\text {equ } \mid \tau=0}=\lambda\left(|y|^{-\gamma} *\left|a_{0}\right|^{2}\right) /(\gamma-1)=-\varphi_{1}
\end{array}\right.
$$

(see Theorem 1.3). We will see later that $\phi_{\text {pha, } 1}$ is the solution to

$$
\left(\begin{array}{l}
\partial_{\tau} b_{\mathrm{pha}, 1}+\nabla \phi_{\mathrm{pha}, 1} \cdot \nabla b_{0}+\nabla \phi_{0} \cdot \nabla b_{\mathrm{pha}, 1}+\frac{1}{2} b_{\mathrm{pha}, 1} \Delta \phi_{0}+\frac{1}{2} b_{0} \Delta \phi_{\mathrm{pha}, 1}=0 \\
\partial_{\tau} \phi_{\mathrm{pha}, 1}+\nabla \phi_{0} \cdot \nabla \phi_{\mathrm{pha}, 1}+\lambda \tau^{\gamma-2}\left(|y|^{-\gamma} * 2 \operatorname{Re} \bar{b}_{0} b_{\mathrm{pha}, 1}\right)=0 \\
b_{\mathrm{pha}, 1 \mid \tau=0} \equiv 0, \quad \phi_{\mathrm{pha}, 1 \mid \tau=0}=\lambda\left(|y|^{-\gamma} *\left|a_{0}\right|^{2}\right) /(\gamma-1)=-\varphi_{1}
\end{array}\right.
$$

(see Remark 5.3). Let $\phi_{\text {equ }}$ be a solution to (1.18)-(1.19). One can check from above systems that $\widetilde{\phi}_{\text {equ }}=\phi_{\text {equ }}+\phi_{\text {pha, } 1}$. Therefore, the modified correction term $\widetilde{\phi}_{\text {equ }}$ is nothing but the superposition of the (usual) correction from equation $\phi_{\text {equ }}$ and the correction from phase $\phi_{\text {pha, } 1}$.

\section{Preliminary results.}

3.1. Properties of the $Y_{p, q}^{s}\left(\mathbb{R}^{n}\right)$ space. We first collect some facts about the space $Y_{p, q}^{s}$ defined in (1.16)-(1.17) (see, also $[1,9]$ ). 
1. $Y_{p, q}^{s_{1}} \subset Y_{p, q}^{s_{2}}$ if $s_{1} \geqslant s_{2}$.

2. $Y_{p, q}^{s}=Y_{p,\left[\min \left(q, 2^{*}\right), \infty\right]}^{s}$ and so $Y_{p, q_{1}}^{s} \subset Y_{p, q_{2}}^{s}$ if $q_{1} \leqslant q_{2}$.

3. If $q<n$ then $Y_{p, q}^{s}=Y_{\left[\min \left(p, q^{*}\right), \infty\right], q}^{s}$. It implies $Y_{p_{1}, q}^{s} \subset Y_{p_{2}, q}^{s}$ for $p_{1} \leqslant p_{2}$ under $q<n$. In particular, $Y_{p, q}^{s} \subset Y_{\left[2^{* *}, \infty\right],\left[2^{*}, \infty\right]}^{s}$ if $n \geqslant 5$, where $2^{* *}:=\left(2^{*}\right)^{*}=$ $2 n /(n-4)$.

4. If $n \geqslant 3$ then any function $f \in X^{s}$ is written uniquely as $f=g+c$, where $g \in Y_{\infty, 2}^{s}\left(=Y_{2^{*}, 2}^{s}\right)$ and $c$ is a constant.

The first property is obvious by definition. The others follow from the following lemma which is a consequence of the Hardy-Littlewood-Sobolev inequality and found in [19, Th. 4.5.9] or [16, Lemma 7]:

LEMmA 3.1. If $\varphi \in \mathcal{D}^{\prime}\left(\mathbb{R}^{n}\right)$ is such that $\nabla \varphi \in L^{p}\left(\mathbb{R}^{n}\right)$ for $\left.p \in\right] 1, n[$, then there exists a constant $c$ such that $\varphi-c \in L^{q}\left(\mathbb{R}^{n}\right)$, with $1 / p=1 / q+1 / n$.

We take a function $f \in Y_{p, q}^{s}$. Then, the indices $p$ and $q$ almost indicate the decay rates at spacial infinity of the function $f$ and its first derivative $\nabla f$, respectively. The second property means that $\nabla f$ is always bounded and decays at the spacial infinity so fast that $\nabla f \in L^{2^{*}}$. What the third property says is that $f$ has a similar decay property. It can be said from the fourth property that $Y_{\infty, 2}^{s}=X^{s}$ in a sense, provided $n \geqslant 3$. Note that every $g \in Y_{\infty, 2}^{s}$ satisfies $g \rightarrow 0$ as $|x| \rightarrow \infty$ by definition (1.16)-(1.17). On the other hand, elements of $X^{s}$ do not necessarily tend to zero at the spacial infinity. We also note that if $q=2$ then we have another definition of $Y_{p, 2}^{s}$ :

$$
Y_{p, 2}^{s}\left(\mathbb{R}^{n}\right)=L^{p}\left(\mathbb{R}^{n}\right) \cap X^{s}\left(\mathbb{R}^{n}\right)
$$

which makes sense for $s>n / 2$.

3.2. Basic existence and approximation results. Operate $\nabla$ to the equation for $\phi^{h}$ in (1.11) and put $w^{h}:=\nabla \phi^{h}$. For our further application, we generalize the system slightly. Let

$$
\begin{aligned}
Q_{1}(a, v) & =-(v \cdot \nabla) a-\frac{1}{2} a \nabla \cdot v, \\
Q_{2}\left(v_{1}, v_{2}\right) & =-\left(v_{1} \cdot \nabla\right) v_{2}, \\
Q_{3}\left(a_{1}, a_{2}\right) & =-\lambda \nabla\left(|x|^{-\gamma} *\left(a_{1} \overline{a_{2}}\right)\right),
\end{aligned}
$$

and consider a system of the following form:

$$
\begin{gathered}
\left\{\begin{array}{c}
\partial_{t} b^{h}=c_{1}^{h} Q_{1}\left(b^{h}, w^{h}\right)+Q_{1}\left(B_{1}^{h}, w^{h}\right)+Q_{1}\left(b^{h}, W_{1}^{h}\right)+R_{1}^{h}+i r^{h} \Delta b^{h}, \\
\partial_{t} w^{h}=c_{2}^{h} Q_{2}\left(w^{h}, w^{h}\right)+Q_{2}\left(W_{2}^{h}, w^{h}\right)+Q_{2}\left(w^{h}, W_{2}^{h}\right) \\
+f^{h}(t)\left(c_{2}^{h} Q_{3}\left(b^{h}, b^{h}\right)+Q_{3}\left(B_{2}^{h}, b^{h}\right)+Q_{3}\left(b^{h}, B_{2}^{h}\right)\right)+R_{2}^{h}, \\
b_{\mid t=0}^{h}=b_{0}^{h}, \quad w_{\mid t=0}^{h}=w_{0}^{h},
\end{array}\right.
\end{gathered}
$$

where $b^{h}$ takes complex value and $w^{h}$ takes real value. Other notation will be made precise in Assumptions 3.2 and 3.3, below. In [9], the existence of a unique solution for this kind of system is shown with explicit coefficients. We shall summarize the parallel result.

Assumption 3.2 (initial data). Let $n \geqslant 3$ and $\max (n / 2-2,0)<\gamma \leqslant n-2$. We suppose the following conditions with some $s>n / 2+1$ : The initial amplitude 
$b_{0}^{h} \in H^{s}\left(\mathbb{R}^{n}\right)$ and the initial velocity $w_{0}^{h} \in Y_{q_{0}, 2}^{s+1}\left(\mathbb{R}^{n}\right)$ for some $\left.q_{0} \in\right] n /(\gamma+1), n[$, uniformly for $h \in[0,1]$, that is, there exists a constant $C$ independent of $h$ such that $\left\|b_{0}^{h}\right\|_{H^{s}}+\left\|w_{0}\right\|_{Y_{q_{0}, 2}^{s+1}\left(\mathbb{R}^{n}\right)} \leqslant C$.

Assumption 3.3 (coefficients). Let $c_{1}^{h}$ and $c_{2}^{h}$ be complex constants bounded uniformly in $h$, and let $r^{h}$ be a real constant bounded uniformly in $h$. Suppose for some $T^{*}>0$ and $s>n / 2+1$ that $f^{h}$ is a real-valued function of time and $f^{h} \in L^{1}\left(\left(0, T^{*}\right)\right)$; $B_{i}^{h}$ and $R_{1}^{h}$ are complex-valued functions of spacetime, and $B_{i}^{h} \in L^{1}\left(\left(0, T^{*}\right) ; H^{s+1}\right)$ and $R_{1}^{h} \in L^{1}\left(\left(0, T^{*}\right) ; H^{s}\right) ; W_{i}^{h}$ and $R_{2}^{h}$ are real-valued functions of spacetime, and $W_{i}^{h} \in L^{1}\left(\left(0, T^{*}\right) ; Y_{\infty, 2}^{s+2}\right)$ and $R_{2}^{h} \in L^{1}\left(\left(0, T^{*}\right) ; Y_{q_{0}, 2}^{s+1}\right)$. Moreover, suppose all above functions are bounded in the corresponding norms uniformly with respect to $h$.

Assumption 3.4 (existence of the limit). In addition to Assumptions 3.2 and 3.3, we suppose the existence of limits of all $b_{0}^{h}, w_{0}^{h}, c_{i}^{h}, r^{h}, f^{h}, B_{i}^{h}, W_{i}^{h}$, and $R_{i}^{h}$ as $h \rightarrow 0$ in the corresponding strong topologies. These strong limits are denoted by $b_{0}$, $w_{0}, c_{i}, r, f, B_{i}, W_{i}$, and $R_{i}$, respectively.

Proposition 3.5. Let Assumptions 3.2 and 3.3 be satisfied. Then, there exists $T>0$ independent of $h, s$, and $q_{0}$, such that for all $h \in[0,1]$ the system (3.5)-(3.6) has a unique solution

$$
\left(b^{h}, w^{h}\right) \in C\left([0, T] ; H^{s} \times Y_{q_{0}, 2}^{s+1}\right) .
$$

Moreover, the norm of $\left(b^{h}, w^{h}\right)$ is bounded uniformly for $h \in[0,1]$. If, in addition, Assumption 3.4 is satisfied, then the pair $\left(b^{h}, w^{h}\right)$ converges to $(b, w):=\left(b^{h}, w^{h}\right)_{\mid h=0}$ in $C\left([0, T] ; H^{s-2} \times Y_{q_{0}, 2}^{s-1}\right)$ as $h \rightarrow 0$. Furthermore, $(b, w)$ solves

$$
\left\{\begin{array}{c}
\partial_{t} b=c_{1} Q_{1}(b, w)+Q_{1}\left(B_{1}, w\right)+Q_{1}\left(b, W_{1}\right)+R_{1}+i r \Delta b \\
\partial_{t} w= \\
c_{2} Q_{2}(w, w)+Q_{2}\left(W_{2}, w\right)+Q_{2}\left(w, W_{2}\right) \\
+f(t)\left(c_{2} Q_{3}(b, b)+Q_{3}\left(B_{2}, b\right)+Q_{3}\left(b, B_{2}\right)\right)+R_{2} \\
b_{\mid t=0}=b_{0}, \quad w_{\mid t=0}=w_{0} .
\end{array}\right.
$$

Proof. The key is the following energy estimate for $s>n / 2+1$

$$
\begin{aligned}
\frac{d}{d t} E^{h} \leqslant & C\left(1+\left|f^{h}(t)\right|\right)\left[\left(\left|c_{1}^{h}\right|+\left|c_{2}^{h}\right|\right)\left(E^{h}\right)^{\frac{3}{2}}\right. \\
& \left.+\left(\left\|B_{1}^{h}\right\|_{H^{s+1}}+\left\|B_{2}^{h}\right\|_{H^{s}}+\left\|\nabla W_{1}^{h}\right\|_{H^{s}}+\left\|\nabla W_{2}^{h}\right\|_{H^{s+1}}\right) E^{h}\right] \\
& +C\left(\left\|R_{1}^{h}\right\|_{H^{s}}+\left\|\nabla R_{2}^{h}\right\|_{H^{s}}\right)\left(E^{h}\right)^{\frac{1}{2}}
\end{aligned}
$$

where $E^{h}:=\left\|b^{h}\right\|_{H^{s}}^{2}+\left\|\nabla w^{h}\right\|_{H^{s}}^{2}$. For more details, see the proof of Proposition 4.1 in [9].

REMARK 3.6. We intend to apply this proposition to the system (1.11). In that case, $f^{h}(t)$ corresponds to $t^{\gamma-2}$, which is singular at $t=0$ if $\gamma<2$. Since $f^{h}(t)$ is only supposed to be integrable, we will see that the system (1.11) (and so the equation (1.8)) has a unique solution for $\gamma>1$ while the singularity. We also note that this corresponds to the fact that the Hartree nonlinearity is short range when $\gamma>1$. 
REMARK 3.7. In the convergence part of Proposition 3.5, it can happen that $n / 2+1 \geqslant s-1>n / 2$. In this case, we use the definition (3.1) instead of (1.16).

We conclude this section with a lemma which we use for the construction of a function $\phi^{h}$ from the corresponding solution $w^{h}=\nabla \phi^{h}$ to (3.5)-(3.6). This lemma is a consequence of Lemma 3.1.

Lemma 3.8. If $\varphi$ satisfies $|\varphi| \rightarrow 0$ as $|x| \rightarrow \infty$ and $\nabla \varphi \in Y_{q, 2}^{s}$ for some $s>n / 2$ and some $q<n, q \leqslant 2^{*}$ then $\varphi \in Y_{q^{*}, q}^{s+1}$.

\section{Proof of Theorem 1.3.}

4.1. Strategy. In this section, we illustrate the strategy of the proof of Theorem 1.3 rather precisely. As in Section 1, we first introduce the semiclassical conformal transform:

$$
u^{\varepsilon}(t, x)=\frac{1}{(1-t)^{n / 2}} \psi^{\varepsilon}\left(\frac{\varepsilon^{\frac{\alpha}{\gamma}}}{1-t}, \frac{x}{1-t}\right) \exp \left(i \frac{|x|^{2}}{2 \varepsilon(t-1)}\right) .
$$

Putting $\tau:=\varepsilon^{\frac{\alpha}{\gamma}} /(1-t)$ and $y:=x /(1-t)$, we find that (1.3)-(1.4) becomes

$$
i \varepsilon^{1-\frac{\alpha}{\gamma}} \partial_{\tau} \psi^{\varepsilon}+\frac{\left(\varepsilon^{1-\frac{\alpha}{\gamma}}\right)^{2}}{2} \Delta_{y} \psi^{\varepsilon}=\lambda \tau^{\gamma-2}\left(|y|^{-\gamma} *\left|\psi^{\varepsilon}\right|^{2}\right) \psi^{\varepsilon}, \quad \psi_{\mid \tau=\varepsilon^{\alpha / \gamma}}^{\varepsilon}=a_{0} .
$$

Put $h=\varepsilon^{1-\alpha / \gamma}$ and denote $\psi^{\varepsilon}$ by $\psi^{h}$. We note that $\varepsilon \rightarrow 0$ is equivalent to $h \rightarrow 0$ as long as $\alpha<\gamma$. Thus, our problem is reduced to the limit $h \rightarrow 0$ of the solution to

$$
i h \partial_{\tau} \psi^{h}+\frac{h^{2}}{2} \Delta \psi^{h}=\lambda \tau^{\gamma-2}\left(|y|^{-\gamma} *\left|\psi^{h}\right|^{2}\right) \psi^{h}, \quad \psi_{\mid \tau=h}^{h} \frac{\alpha}{\gamma-\alpha}(y)=a_{0}(y) .
$$

Our strategy is to seek a solution $\psi^{h}$ to (1.8) represented as

$$
\psi^{h}(\tau, y)=a^{h}(\tau, y) e^{i \phi^{h}(\tau, y) / h},
$$

with a complex-valued space-time function $a^{h}$ and a real-valued space-time function $\phi^{h}$. Note that $a^{h}$ is expected to be complex-valued, even if its initial value $a_{0}$ is real-valued. Substituting the form (1.10) into (1.8), we obtain

$$
\begin{aligned}
-a^{h}\left(\partial_{\tau} \phi^{h}+\frac{1}{2}\left|\nabla \phi^{h}\right|^{2}+\right. & \left.\lambda \tau^{\gamma-2}\left(|y|^{-\gamma} *\left|a^{h}\right|^{2}\right)\right) \\
& +i h\left(\partial_{\tau} a^{h}+\left(\nabla \phi^{h} \cdot \nabla\right) a^{h}+\frac{1}{2} a^{h} \Delta \phi^{h}-i \frac{h}{2} \Delta a^{h}\right)=0 .
\end{aligned}
$$

To obtain a solution of the above equation (hence, of (1.8)), we choose to consider

$$
\left\{\begin{array}{l}
\partial_{\tau} a^{h}+\nabla \phi^{h} \cdot \nabla a^{h}+\frac{1}{2} a^{h} \Delta \phi^{h}=i \frac{h}{2} \Delta a^{h}, \\
\partial_{\tau} \phi^{h}+\frac{1}{2}\left|\nabla \phi^{h}\right|^{2}+\lambda \tau^{\gamma-2}\left(|y|^{-\gamma} *\left|a^{h}\right|^{2}\right)=0, \\
a_{\mid \tau=h \frac{\alpha}{\gamma-\alpha}}^{h}=a_{0}, \quad \phi_{\mid \tau=h}^{h} \frac{\alpha}{\gamma-\alpha}=0 .
\end{array}\right.
$$

The point is that this system can be regarded as a symmetric hyperbolic system with semilinear perturbation. In Section 4.2, we first prove that it admits a unique solution 
with suitable regularity (see Proposition 4.1), hence providing a solution to (1.8) and (1.3)-(1.4).

By (1.10), in order to obtain a leading order WKB type approximate solution it suffices to determine $O\left(h^{0}\right)$ and $O\left(h^{1}\right)$ terms of $\phi^{h}$ in the limit $h \rightarrow 0$. Letting $h=0$ in (1.11), we formally obtain the $O\left(h^{0}\right)$ term $\left(b_{0}, \phi_{0}\right)$ which solves

$$
\left\{\begin{array}{l}
\partial_{t} b_{0}+\nabla \phi \cdot \nabla b_{0}+\frac{1}{2} b_{0} \Delta \phi_{0}=0, \\
\partial_{t} \phi_{0}+\frac{1}{2}\left|\nabla \phi_{0}\right|^{2}+\lambda t^{\gamma-2}\left(|y|^{-\gamma} *\left|b_{0}\right|^{2}\right)=0, \\
b_{0 \mid \tau=0}=a_{0}, \quad \phi_{0 \mid \tau=0}=0
\end{array}\right.
$$

introduced in Section 1. The difficulty of finding $h^{1}$-terms lies in the following two respects; firstly, the equation (1.11) depends on $h$ through the term $i \frac{h}{2} \Delta a^{h}$; and secondly the initial data of (1.11) is moving at a speed $h^{\frac{\alpha}{\gamma-\alpha}}$. In Section 4.3, we give the time expansion of $\left(b_{0}, \phi_{0}\right)$ around $t=0$. We will obtain an expansion of the form

$$
b_{0}(\tau, y) \asymp \sum_{j=0}^{\infty} \tau^{\gamma j} a_{j}(y), \quad \phi_{0}(\tau, y) \asymp \sum_{j=1}^{\infty} \tau^{\gamma j-1} \varphi_{j}(y)
$$

(Proposition 4.2). This expansion is essential in handling the moving initial-data.

In Section 4.4, we finally determine $O\left(h^{1}\right)$ term in the case $\alpha \geqslant 1$. What to show is the existence of the limits

$$
\frac{a^{h}(\tau)-b_{0}(\tau)}{h} \rightarrow b_{\text {equ }}(\tau), \quad \frac{\phi^{h}(\tau)-\phi_{0}(\tau)}{h} \rightarrow \phi_{\text {equ }}(\tau)
$$

as $h \rightarrow 0$. A formal differentiation of (1.11) with respect to $h$ suggests that $\left(b_{\text {equ }}, \phi_{\text {equ }}\right)$ may solve the linearized system

$$
\left(\begin{array}{l}
\partial_{\tau} b_{\text {equ }}+\nabla \phi_{\text {equ }} \cdot \nabla b_{0}+\nabla \phi_{0} \cdot \nabla b_{\text {equ }}+\frac{1}{2} b_{\text {equ }} \Delta \phi_{0}+\frac{1}{2} b_{0} \Delta \phi_{\text {equ }}=\frac{i}{2} \Delta b_{0} \\
\partial_{\tau} \phi_{\text {equ }}+\nabla \phi_{0} \cdot \nabla \phi_{\text {equ }}+\lambda \tau^{\gamma-2}\left(|x|^{-\gamma} * 2 \operatorname{Re} \overline{b_{0}} b_{\text {equ }}\right)=0
\end{array}\right.
$$

By means of (4.1), the following estimates hold at the initial time:

$$
\begin{aligned}
& \frac{a^{h}\left(h^{\frac{\alpha}{\gamma-\alpha}}\right)-b_{0}\left(h^{\frac{\alpha}{\gamma-\alpha}}\right)}{h}=-\frac{b_{0}\left(h^{\frac{\alpha}{\gamma-\alpha}}\right)-a_{0}}{h}=O\left(h^{\frac{\alpha \gamma}{\gamma-\alpha}-1}\right), \\
& \frac{\phi^{h}\left(h^{\frac{\alpha}{\gamma-\alpha}}\right)-\phi_{0}\left(h^{\frac{\alpha}{\gamma-\alpha}}\right)}{h}=-\frac{\phi_{0}\left(h^{\frac{\alpha}{\gamma-\alpha}}\right)}{h}=O\left(h^{\frac{\alpha(\gamma-1)}{\gamma-\alpha}-1}\right) .
\end{aligned}
$$

Note that $\frac{\alpha \gamma}{\gamma-\alpha}>1$ for all $\alpha \geqslant 1$, however, $\frac{\alpha(\gamma-1)}{\gamma-\alpha}>1$ if $\alpha>1$ and $\frac{\alpha(\gamma-1)}{\gamma-\alpha}=1$ if $\alpha=1$. In particular,

$$
\frac{\phi^{h}\left(h^{\frac{\alpha}{\gamma-\alpha}}\right)-\phi_{0}\left(h^{\frac{\alpha}{\gamma-\alpha}}\right)}{h} \rightarrow \begin{cases}0 & \text { if } \alpha>1, \\ -\varphi_{1} & \text { if } \alpha=1,\end{cases}
$$

where $\varphi_{1}$ is defined in (4.1). Therefore, the $O\left(h^{1}\right)$ term is described by $\left(b_{\text {equ }}, \phi_{\text {equ }}\right)$ solving (1.18) with (1.19) if $\alpha>1$ and with (1.20) if $\alpha=1$. In Section 5, we consider the case $\alpha<1$. In this case, the above powers $\frac{\alpha \gamma}{\gamma-\alpha}$ and $\frac{\alpha(\gamma-1)}{\gamma-\alpha}$ are less than one, in general. Therefore, there appear several terms which is order less than $O\left(h^{1}\right)$ in the expansion of $\left(a^{h}, \phi^{h}\right)$. We determine all these terms and obtain the asymptotic behavior of $\left(a^{h}, \phi^{h}\right)$ (see, Theorem 5.1).

In Sections 4 and 5, we mainly work with $v^{h}=\nabla \phi^{h}$ instead of $\phi^{h}$ itself. Note that, by means of Lemma 3.8, it is easy to construct $\phi^{h}$ from $v^{h}$. 
4.2. Existence of phase-amplitude form solution. According to the strategy in Section 4.1, we first show that the system (1.11) has a unique solution.

Proposition 4.1. Let Assumption 1.2 be satisfied. Assume $0<\alpha<\gamma$. Then, there exists $T>0$ independent of $h$ such that, for all $h \in(0,1]$, there exists a unique solution $\psi^{h} \in C\left(\left[h^{\frac{\alpha}{\gamma-\alpha}}, T+h^{\frac{\alpha}{\gamma-\alpha}}\right] ; H^{\infty}\right)$ to (1.8). Moreover, $\psi^{h}$ is written as

$$
\psi^{h}=a^{h} e^{i \frac{\phi^{h}}{h}},
$$

where

$$
a^{h} \in C\left(\left[h^{\frac{\alpha}{\gamma-\alpha}}, T+h^{\frac{\alpha}{\gamma-\alpha}}\right] ; H^{\infty}\right) \cap C^{\infty}\left(\left(h^{\frac{\alpha}{\gamma-\alpha}}, T+h^{\frac{\alpha}{\gamma-\alpha}}\right] ; H^{\infty}\right)
$$

and

$$
\begin{aligned}
\phi^{h} \in & C\left(\left[h^{\frac{\alpha}{\gamma-\alpha}}, T+h^{\frac{\alpha}{\gamma-\alpha}}\right] ; Y_{(n / \gamma, \infty],(n /(\gamma+1), \infty]}^{\infty}\right) \\
& \cap C^{\infty}\left(\left(h^{\frac{\alpha}{\gamma-\alpha}}, T+h^{\frac{\alpha}{\gamma-\alpha}}\right] ; Y_{(n / \gamma, \infty],(n /(\gamma+1), \infty]}^{\infty}\right) .
\end{aligned}
$$

Moreover, there exists a limit $\left(b_{0}, \phi_{0}\right):=\left(a^{h}, \phi^{h}\right)_{\mid h=0}$ belonging the same function space as $\left(a^{h}, \phi^{h}\right)(h>0)$, and $\left(a^{h}, \phi^{h}\right)$ converges strongly to $\left(b_{0}, \phi_{0}\right)$ as $h \rightarrow 0$. Furthermore, $\left(b_{0}, \phi_{0}\right)$ solves (1.13).

Proof. We set velocity $v^{h}=\nabla \phi^{h}$. Then, the pair $\left(a^{h}, v^{h}\right)$ solves

$$
\begin{aligned}
& \left\{\begin{array}{l}
\partial_{\tau} a^{h}=Q_{1}\left(a^{h}, v^{h}\right)+i \frac{h}{2} \Delta a^{h}, \\
\partial_{\tau} v^{h}=Q_{2}\left(v^{h}, v^{h}\right)+\tau^{\gamma-2} Q_{3}\left(a^{h}, a^{h}\right),
\end{array}\right. \\
& a_{\mid \tau=h}^{h} \frac{\alpha}{\gamma-\alpha}=a_{0}, \quad v_{\mid \tau=h}^{h} \frac{\alpha}{\gamma-\alpha}=0,
\end{aligned}
$$

where $Q_{1}, Q_{2}$, and $Q_{3}$ are defined by (3.2), (3.3), and (3.4), respectively. To fix the initial time, we employ the time translation $\tau=t+h^{\frac{\alpha}{\gamma-\alpha}}$. Then, the equation is

$$
\begin{aligned}
&\left\{\begin{aligned}
\partial_{t} a^{h} & =Q_{1}\left(a^{h}, v^{h}\right)+i \frac{h}{2} \Delta a^{h}, \\
\partial_{t} v^{h} & =Q_{2}\left(v^{h}, v^{h}\right)+\left(t+h^{\frac{\alpha}{\gamma-\alpha}}\right)^{\gamma-2} Q_{3}\left(a^{h}, a^{h}\right),
\end{aligned}\right. \\
& a_{\mid t=0}^{h}=a_{0}, v_{\mid t=0}^{h}=0 .
\end{aligned}
$$

Now, the assumption $\gamma>1$ implies that $\left(t+h^{\frac{\alpha}{\gamma-\alpha}}\right)^{\gamma-2}$ is integrable over $\left(0, T^{*}\right)$ for some $T^{*}>0$ and its integral is uniformly bounded with respect to $h$. Fix $s>n / 2+1$. Then, applying Proposition 3.5 with $c_{1}^{h}=c_{2}^{h}=1, r^{h}=h / 2, f^{h}(t)=\left(t+h^{\frac{\alpha}{\gamma-\alpha}}\right)^{\gamma-2}$, and $B_{i}^{h} \equiv W_{i}^{h} \equiv R_{i}^{h} \equiv 0$, we obtain the existence time $0<T \leqslant T^{*}$ independent of $\varepsilon$ and the unique solution

$$
\left(a^{h}, v^{h}\right) \in C\left([0, T] ; H^{s} \times Y_{(n /(\gamma+1), \infty], 2}^{s+1}\right)
$$

to (4.4)-(4.5) such that $\left\|a^{h}\right\|_{H^{s}}^{2}+\left\|\nabla v^{h}\right\|_{H^{s}}^{2}$ is bounded uniformly with respect to $t \in[0, T]$. The upper bound depends only on $\left\|a_{0}\right\|_{H^{s}}$. Notice that Assumption 3.2 is satisfied for all $s>n / 2+1$ and $\left.q_{0} \in\right] n /(\gamma+1), n[$. 
By the equation and the Hardy-Littlewood-Sobolev inequality, $\partial_{t} v^{h}$ belongs to $C\left([0, T] ; Y_{(n /(\gamma+1), \infty], 2}^{s}\right)$. Hence, we see from Lemma 3.8 that $\partial_{t} \phi^{h} \in$ $C\left([0, T] ; Y_{(n / \gamma, \infty],(n /(\gamma+1), \infty]}^{s+1}\right)$. Since $\phi_{\mid t=0}^{h}=0$, we also have

$$
\phi^{h} \in C\left([0, T] ; Y_{(n / \gamma, \infty],(n /(\gamma+1), \infty]}^{s+1}\right) .
$$

Therefore, we have $\phi^{h} \rightarrow 0$ as $|x| \rightarrow \infty$. Then, again applying Lemma 3.8 to $v^{h}$, we conclude that $\phi^{h} \in C\left([0, T] ; Y_{(n / \gamma, \infty],(n /(\gamma+1), \infty])}^{s+2}\right.$. Since the existence time $T$ is independent of $s$, we have $a^{h} \in C\left([0, T] ; H^{\infty}\right)$ and $\phi^{h} \in C\left([0, T] ; Y_{(n / \gamma, \infty],(n /(\gamma+1), \infty]}^{\infty}\right)$. The bootstrap argument gives the $C^{\infty}$ regularity with respect time. The existence of the limit $\left(b_{0}, \phi_{0}\right)$ and the convergence $\left(a^{h}, \phi^{h}\right) \rightarrow\left(b_{0}, \phi_{0}\right)$ as $h \rightarrow 0$ follow from the latter part of Proposition 3.5 and Lemma 3.8.

4.3. Time expansion of the limit solution near $\tau=0$. By Proposition 4.1, the system (4.2)-(4.3) has a unique solution even if $h=0$. We keep working with $v^{h}=\nabla \phi^{h}$ instead of $\phi^{h}$ Write $\left(b_{0}, w_{0}\right):=\left(a^{h}, v^{h}\right)_{\mid h=0}$. The main difficulty of describing the asymptotic behavior of $\left(a^{h}, v^{h}\right)$ as $h \rightarrow 0$ comes from the fact that the initial data is given at $\tau=h^{\frac{\alpha}{\gamma-\alpha}}$. In order to handle this $h$-dependence of the initial time, we give a time expansion of $\left(b_{0}, w_{0}\right)$ around $\tau=0$.

Note that $\left(b_{0}, w_{0}\right)$ solves

$$
\begin{array}{cc}
\partial_{\tau} b_{0}=Q_{1}\left(b_{0}, w_{0}\right), & \partial_{\tau} w_{0}=Q_{2}\left(w_{0}, w_{0}\right)+\tau^{\gamma-2} Q_{3}\left(b_{0}, b_{0}\right), \\
b_{0 \mid \tau=0}=a_{0}, & w_{0 \mid \tau=0}=0,
\end{array}
$$

where the quadratic forms $Q_{i}$ are defined by (3.2)-(3.4).

Proposition 4.2. Let $\left(b_{0}, w_{0}\right)=\left(b_{0}, \nabla \phi_{0}\right)$ be the unique solution to $(4.6)-(4.7)$ defined by Proposition 4.1. Then, it holds that

$$
\begin{aligned}
b_{0}(\tau, y) & =\sum_{j=0}^{J} \tau^{\gamma j} a_{j}(y)+o\left(\tau^{\gamma J}\right) \quad \text { in } H^{\infty} \\
w_{0}(\tau, y) & =\sum_{j=1}^{J} \tau^{\gamma j-1} v_{j}(y)+o\left(\tau^{\gamma J-1}\right) \quad \text { in } Y_{(n /(\gamma+1), \infty], 2}^{\infty}
\end{aligned}
$$

as $\tau \rightarrow 0$ for all $J$, where $a_{0}$ is the initial data for $b_{0}, a_{j}$ and $v_{j}$ are defined by

$$
a_{j}=\frac{1}{\gamma j} \sum_{k_{1} \geqslant 0, k_{2} \geqslant 1, k_{1}+k_{2}=j} Q_{1}\left(a_{k_{1}}, v_{k_{2}}\right)
$$

for $j \geqslant 1, v_{1}=Q_{3}\left(a_{0}, a_{0}\right) /(\gamma-1)$, and

$$
v_{j}=\frac{1}{\gamma j-1}\left[\sum_{k_{1} \geqslant 1, k_{2} \geqslant 1, k_{1}+k_{2}=j} Q_{2}\left(v_{k_{1}}, v_{k_{2}}\right)+\sum_{k_{1} \geqslant 0, k_{2} \geqslant 0, k_{1}+k_{2}=j-1} Q_{3}\left(a_{k_{1}}, a_{k_{2}}\right)\right]
$$

for $j \geqslant 2$ with the quadratic forms $Q_{i}$ defined by (3.2)-(3.4). Moreover, $\phi_{0}$ is expanded as

$$
\phi_{0}(\tau, y)=\sum_{j=1}^{J} \tau^{\gamma j-1} \varphi_{j}(y)+o\left(\tau^{\gamma J-1}\right) \quad \text { in } Y_{(n / \gamma, \infty],(n /(\gamma+1), \infty]}^{\infty}
$$


as $\tau \rightarrow 0$ for all $J \geqslant 1$, where $\varphi_{j}$ is given by $\varphi_{1}=\frac{\lambda}{1-\gamma}\left(|y|^{-\gamma} *\left|a_{0}\right|^{2}\right)$ and

$$
\begin{aligned}
& \varphi_{j}=\frac{1}{1-\gamma j}\left[\sum_{k_{1} \geqslant 1, k_{2} \geqslant 1, k_{1}+k_{2}=j} \frac{1}{2}\left(\nabla \varphi_{k_{1}} \cdot \nabla \varphi_{k_{2}}\right)\right. \\
&+\sum_{k_{1} \geqslant 0, k_{2} \geqslant 0, k_{1}+k_{2}=j-1} \lambda\left(|y|^{-\gamma} *\left(a_{k_{1}} \overline{a_{k_{2}}}\right)\right] .
\end{aligned}
$$

REMARK 4.3. Using the “こ" sign defined in Notation 1.1, the above three expansions (4.8), (4.9), and (1.24) can be written as $b_{0}(\tau) \asymp \sum_{j=0}^{J} \tau^{\gamma j} a_{j}$ in $H^{\infty}, w_{0}(\tau) \asymp$ $\sum_{j=1}^{J} \tau^{\gamma j-1} v_{j}$ in $Y_{(n /(\gamma+1), \infty], 2}^{\infty}$, and $\phi_{0}(\tau) \asymp \sum_{j=1}^{J} \tau^{\gamma j-1} \varphi_{j}$ in $Y_{(n / \gamma, \infty],(n /(\gamma+1), \infty]}^{\infty}$, respectively that

Proof. We first note that, by the definitions of $Q_{i}$, it follows for all $s>n / 2+1$

$$
\begin{aligned}
& \left\|Q_{1}(b, v)\right\|_{H^{s}} \leqslant C_{s}\|b\|_{H^{s+1}}\|v\|_{Y_{(n /(\gamma+1), \infty], 2}^{s+1}}, \\
& \left\|Q_{2}\left(v_{1}, v_{2}\right)\right\|_{Y_{(n /(\gamma+1), \infty], 2}^{s}} \leqslant C_{s}\left\|v_{1}\right\|_{Y_{(n /(\gamma+1), \infty], 2}^{s}}\left\|v_{2}\right\|_{Y_{(n /(\gamma+1), \infty], 2}^{s+1}}, \\
& \left\|Q_{3}\left(b_{1}, b_{2}\right)\right\|_{Y_{(n /(\gamma+1), \infty], 2}^{s}} \leqslant C_{s}\left\|b_{1}\right\|_{H^{s}}\left\|b_{2}\right\|_{H^{s}} .
\end{aligned}
$$

Therefore, we see that $a_{l}$ and $v_{l}$ are bounded in $H^{\infty}$ and $Y_{(n /(\gamma+1), \infty], 2}^{\infty}$, respectively. For simplicity, in this proof we denote $Y_{(n /(\gamma+1), \infty], 2}^{s}$ by $Y^{s}$. Denote $\sum_{j=0}^{l} \tau^{\gamma j} a_{j}$ and $\sum_{j=1}^{l} \tau^{\gamma j-1} v_{j}$ by $\widetilde{a}_{l}$ and $\widetilde{v}_{l}$, respectively. Then, it suffices to show that

$$
\begin{array}{ll}
\left\|b_{0}-\widetilde{a}_{l}\right\|_{L^{\infty}\left([0, \tau] ; H^{\infty}\right)}=o\left(\tau^{\gamma l}\right) & \forall l \geqslant 0, \\
\left\|w_{0}-\widetilde{v}_{l}\right\|_{L^{\infty}\left([0, \tau] ; Y^{\infty}\right)}=o\left(\tau^{\gamma l-1}\right) & \forall l \geqslant 1 .
\end{array}
$$

Step 1. Since $b_{0} \in C\left([0, T] ; H^{\infty}\right)$ and $b_{0}(0)=a_{0}=\widetilde{a}_{0},(4.10)$ is trivial if $l=0$. We show (4.11) for $l=1$. By the second equation of (4.6), it holds for $s>n / 2+1$ that

$$
\begin{aligned}
\left\|w_{0}(\tau)\right\|_{Y^{s}} & \leqslant C_{1} \int_{0}^{\tau}\left\|w_{0}(t)\right\|_{Y^{s}} d t+C_{2} \int_{0}^{\tau} t^{\gamma-2} d t \\
& \leqslant C_{1} \tau\left\|w_{0}\right\|_{L^{\infty}\left((0, \tau] ; Y^{s}\right)}+C_{2}^{\prime} \tau^{\gamma-1}
\end{aligned}
$$

where $C_{1}$ depends on $s$ and $C\left([0, T] ; Y^{\infty}\right)$ norm of $w_{0}$, and $C_{2}$ depends on $s$ and $C\left([0, T] ; H^{\infty}\right)$ norm of $b_{0}$. The right hand side is monotone increasing in time, hence this gives

$$
\left\|w_{0}\right\|_{L^{\infty}\left((0, \tau] ; Y^{s}\right)} \leqslant C_{1} \tau\left\|w_{0}\right\|_{L^{\infty}\left((0, t] ; Y^{s}\right)}+C_{2}^{\prime} \tau^{\gamma-1}
$$

Choose $\tau$ so small that $C_{1} \tau \leqslant 1 / 2$. Then, we obtain

$$
\left\|w_{0}\right\|_{L^{\infty}\left((0, \tau] ; Y^{\infty}\right)}=O\left(\tau^{\gamma-1}\right)
$$

since $s>n / 2+1$ is arbitrary. Again by the equation, it holds that

$$
w_{0}-\widetilde{v}_{1}=\int_{0}^{\tau} Q_{2}\left(w_{0}, w_{0}\right) d t+\int_{0}^{\tau} t^{\gamma-2}\left(Q_{3}\left(b_{0}, b_{0}-a_{0}\right)+Q_{3}\left(b_{0}-a_{0}, a_{0}\right)\right) d t .
$$


Since $w_{0}$ is order $O\left(\tau^{\gamma-1}\right)$ in $L^{\infty}\left((0, \tau] ; Y^{\infty}\right)$, the first integral of the right hand side is order $O\left(\tau^{2 \gamma-1}\right)$ in $L^{\infty}\left((0, \tau] ; Y^{\infty}\right)$. Similarly, the fact that $b_{0}-a_{0}$ is order $o(1)$ in $L^{\infty}\left((0, \tau] ; H^{\infty}\right)$ shows the second integral is order $o\left(\tau^{\gamma-1}\right)$ in $L^{\infty}\left((0, \tau] ; Y^{\infty}\right)$, which proves (4.11) for $l=1$.

Step 2. We prove (4.10) and (4.11) for large $l$ by induction. By the definition of $a_{j}$, an explicit calculation shows

$$
\begin{aligned}
\partial_{\tau} b_{0}= & Q_{1}\left(b_{0}, w_{0}\right)=Q_{1}\left(b_{0}, w_{0}-\widetilde{v}_{1}\right)+Q_{1}\left(b_{0}-\widetilde{a}_{0}, \tau^{\gamma-1} v_{1}\right)+\partial_{\tau}\left(\tau^{\gamma} a_{1}\right) \\
= & Q_{1}\left(b_{0}, w_{0}-\widetilde{v}_{2}\right)+Q_{1}\left(b_{0}-\widetilde{a}_{0}, \tau^{2 \gamma-1} v_{2}\right)+Q_{1}\left(b_{0}-\widetilde{a}_{1}, \tau^{\gamma-1} v_{1}\right) \\
& +\partial_{\tau}\left(\tau^{\gamma} a_{1}+\tau^{2 \gamma} a_{2}\right)=\cdots \\
= & Q_{1}\left(b_{0}, w_{0}-\widetilde{v}_{l}\right)+\sum_{l_{1}=0}^{l-1} Q_{1}\left(b_{0}-\widetilde{a}_{l_{1}}, \tau^{\gamma\left(l-l_{1}\right)-1} v_{l-l_{1}}\right)+\partial_{\tau}\left(\sum_{j=1}^{l} \tau^{\gamma j} a_{j}\right) .
\end{aligned}
$$

Similarly, it holds that

$$
\begin{aligned}
\partial_{\tau} w_{0}= & Q_{2}\left(w_{0}, w_{0}\right)+\tau^{\gamma-2} Q_{3}\left(b_{0}, b_{0}\right)=\cdots \\
= & Q_{2}\left(w_{0}, w_{0}-\widetilde{v}_{l}\right)+\sum_{l_{1}=1}^{l} Q_{2}\left(w_{0}-\widetilde{v}_{l_{1}}, \tau^{\gamma\left(l-l_{1}+1\right)-1} v_{l-l_{1}+1}\right) \\
& +\tau^{\gamma-2}\left(Q_{3}\left(b_{0}, b_{0}-\widetilde{a}_{l}\right)+\sum_{l_{1}=0}^{l} Q_{3}\left(b_{0}-\widetilde{a}_{l_{1}}, \tau^{\gamma\left(l-l_{1}\right)} a_{l-l_{1}}\right)\right) \\
& +\partial_{\tau}\left(\sum_{j=1}^{l+1} \tau^{\gamma j-1} v_{j}\right) .
\end{aligned}
$$

Integrating these identities with respect to time, we obtain

$$
b_{0}-\widetilde{a}_{l}=\int_{0}^{\tau}\left(Q_{1}\left(b_{0}, w_{0}-\widetilde{v}_{l}\right)+\sum_{l_{1}=0}^{l-1} Q_{1}\left(b_{0}-\widetilde{a}_{l_{1}}, t^{\gamma\left(l-l_{1}\right)-1} v_{l-l_{1}}\right)\right) d t
$$

and

$$
\begin{aligned}
w_{0}-\widetilde{v}_{l+1}= & \int_{0}^{\tau}\left(Q_{2}\left(w_{0}, w_{0}-\widetilde{v}_{l}\right)+\sum_{l_{1}=1}^{l} Q_{2}\left(w_{0}-\widetilde{v}_{l_{1}}, t^{\gamma\left(l-l_{1}+1\right)-1} v_{l-l_{1}+1}\right)\right) d t \\
& +\int_{0}^{\tau} t^{\gamma-2}\left(Q_{3}\left(b_{0}, b_{0}-\widetilde{a}_{l}\right)+\sum_{l_{1}=0}^{l} Q_{3}\left(b_{0}-\widetilde{a}_{l_{1}}, t^{\gamma\left(l-l_{1}\right)} a_{l-l_{1}}\right)\right) d t
\end{aligned}
$$

Now, let $L \geqslant 1$ be an integer. If (4.10) holds for $l \leqslant L-1$ and (4.11) holds for $l \leqslant L$, then we see that (4.12) gives (4.10) with $l=L$. On the other hand, if both (4.10) and (4.11) hold for $l \leqslant L$, then we obtain (4.11) with $l=L+1$ from (4.13).

The expansion of $\phi_{0}$ is an immediate consequence of the expansion of $w_{0}=\nabla \phi_{0}$. Since

$$
Q_{2}\left(v_{k_{1}}, v_{k_{2}}\right)+Q_{2}\left(v_{k_{2}}, v_{k_{1}}\right)=-\nabla\left(v_{k_{1}} \cdot v_{k_{2}}\right)=-\nabla\left(\frac{1}{2} v_{k_{1}} \cdot v_{k_{2}}+\frac{1}{2} v_{k_{2}} \cdot v_{k_{1}}\right),
$$


we deduce from the definition $v_{j}$ that

$$
\begin{aligned}
\nabla \varphi_{j}=v_{j}=\frac{1}{1-\gamma j} \nabla\left[\sum_{k_{1} \geqslant 1, k_{2} \geqslant 1, k_{1}+k_{2}=j}\right. & \frac{1}{2}\left(\nabla \varphi_{k_{1}} \cdot \nabla \varphi_{k_{2}}\right) \\
& +\sum_{k_{1} \geqslant 0, k_{2} \geqslant 0, k_{1}+k_{2}=j-1} \lambda\left(|y|^{-\gamma} *\left(a_{k_{1}} \overline{a_{k_{2}}}\right)\right] .
\end{aligned}
$$

By Lemma $3.8, \varphi_{j}$ belongs to $Y_{(n / \gamma, \infty],(n /(\gamma+1), \infty]}^{\infty}$. $\square$

4.4. Asymptotic behavior of the phase-amplitude form solution. The following proposition completes the proof of the theorem.

Proposition 4.4. Let Assumption 1.2 satisfied and $\alpha \geqslant 1$. Let $T>0$ and $\left(a^{h}, v^{h}\right)$ be as in Proposition 4.1. Let $\left(b_{0}, w_{0}\right):=\left(a^{h}, v^{h}\right)_{\mid h=0}$. Then, there exists $\left(b_{\text {equ }}, w_{\text {equ }}\right) \in C\left([0, T] ; H^{\infty} \times Y_{(n /(\gamma+1), \infty], 2}^{\infty}\right)$ such that the following asymptotics holds:

$$
\begin{aligned}
& a^{h}(\tau)=b_{0}(\tau)+h b_{\text {equ }}\left(\tau-h^{\frac{\alpha}{\gamma-\alpha}}\right)+o(h) \quad \text { in } C\left(\left[h^{\frac{\alpha}{\gamma-\alpha}}, T\right] ; H^{\infty}\right), \\
& v^{h}(\tau)=w_{0}(\tau)+h w_{\text {equ }}\left(\tau-h^{\frac{\alpha}{\gamma-\alpha}}\right)+o(h) \quad \text { in } C\left(\left[h^{\frac{\alpha}{\gamma-\alpha}}, T\right] ; Y_{(n /(\gamma+1), \infty], 2}^{\infty}\right) .
\end{aligned}
$$

Moreover, $\left(b_{\text {equ }}, w_{\text {equ }}\right)$ solves

$$
\left\{\begin{array}{l}
\partial_{\tau} b_{\text {equ }}=Q_{1}\left(b_{0}, w_{\text {equ }}\right)+Q_{1}\left(b_{\text {equ }}, w_{0}\right)+i \frac{1}{2} \Delta b_{0} \\
\partial_{\tau} w_{\text {equ }}=Q_{2}\left(w_{0}, w_{\text {equ }}\right)+Q_{2}\left(w_{\text {equ }}, w_{0}\right)+\tau^{\gamma-2}\left(Q_{3}\left(b_{0}, b_{\text {equ }}\right)+Q_{3}\left(b_{\text {equ }}, b_{0}\right)\right)
\end{array}\right.
$$

with the data

$$
b_{\mathrm{equ} \mid \tau=0}=0, \quad w_{\mathrm{equ} \mid \tau=0}= \begin{cases}0 & \text { if } \alpha>1, \\ -v_{1} & \text { if } \alpha=1,\end{cases}
$$

where $v_{1}$ is defined in Proposition 4.2.

REMARK 4.5. Since

$$
b_{\text {equ }}\left(\tau-h^{\frac{\alpha}{\gamma-\alpha}}\right)=b_{\text {equ }}(\tau)+o(1), \quad w_{\text {equ }}\left(\tau-h^{\frac{\alpha}{\gamma-\alpha}}\right)=w_{\text {equ }}(\tau)+o(1)
$$

by continuity, (4.14) implies

$$
\begin{aligned}
& a^{h}(\tau)=b_{0}(\tau)+h b_{\text {equ }}(\tau)+o(h) \quad \text { in } C\left(\left[h^{\frac{\alpha}{\gamma-\alpha}}, T\right] ; H^{\infty}\right), \\
& v^{h}(\tau)=w_{0}(\tau)+h w_{\text {equ }}(\tau)+o(h) \quad \text { in } C\left(\left[h^{\frac{\alpha}{\gamma-\alpha}}, T\right] ; Y_{(n /(\gamma+1), \infty], 2}^{\infty}\right) .
\end{aligned}
$$

From this asymptotics and the transforms (1.7) and (1.10), we immediately obtain the asymptotics (1.21).

Proof. Let $\left(a^{h}, v^{h}\right)$ be the solution to $(4.2)-(4.3)$. Let $\left(b_{0}, w_{0}\right)$ be the solution to (4.6)-(4.7). We put

$$
\begin{aligned}
b^{h}(\tau, y) & :=\frac{a^{h}\left(\tau+h^{\frac{\alpha}{\gamma-\alpha}}, y\right)-b_{0}\left(\tau+h^{\frac{\alpha}{\gamma-\alpha}}, y\right)}{h} \\
w^{h}(\tau, y) & :=\frac{v^{h}\left(\tau+h^{\frac{\alpha}{\gamma-\alpha}}, y\right)-w_{0}\left(\tau+h^{\frac{\alpha}{\gamma-\alpha}}, y\right)}{h} .
\end{aligned}
$$


Then, $\left(b^{h}, w^{h}\right)$ solves

$$
\begin{gathered}
\left\{\begin{aligned}
\partial_{\tau} b^{h}= & h Q_{1}\left(b^{h}, w^{h}\right)+Q_{1}\left(b_{0}, w^{h}\right)+Q_{1}\left(b^{h}, w_{0}\right)+i \frac{1}{2} \Delta b_{0}+i \frac{h}{2} \Delta b^{h}, \\
\partial_{\tau} w^{h}= & h Q_{2}\left(w^{h}, w^{h}\right)+Q_{2}\left(w_{0}, w^{h}\right)+Q_{2}\left(w^{h}, w_{0}\right) \\
& +\left(\tau+h^{\frac{\alpha}{\gamma-\alpha}}\right)^{\gamma-2}\left(h Q_{3}\left(b^{h}, b^{h}\right)+Q_{3}\left(b_{0}, b^{h}\right)+Q_{3}\left(b^{h}, b_{0}\right)\right),
\end{aligned}\right. \\
b_{\mid \tau=0}^{h}=\frac{b_{0 \mid \tau=0}-b_{0}\left(h^{\frac{\alpha}{\gamma-\alpha}}\right)}{h}, \quad w_{\mid \tau=0}^{h}=\frac{w_{0 \mid \tau=0}-w_{0}\left(h^{\frac{\alpha}{\gamma-\alpha}}\right)}{h} .
\end{gathered}
$$

We apply Proposition 3.5 with these initial data and $c_{1}^{h}=c_{2}^{h}=h, r^{h}=h / 2, f^{h}(t)=$ $\left(t+h^{\frac{\alpha}{\gamma-\alpha}}\right)^{\gamma-2}, B_{1}^{h}=B_{2}^{h}=b_{0}, W_{1}^{h}=W_{2}^{h}=w_{0}, R_{1}^{h}=(i / 2) \Delta b_{0}$, and $R_{2}^{h}=0$. Note that the initial data (4.18) is uniformly bounded if $\alpha \geqslant 1$ since an application of (4.8) and (4.9) gives

$$
\begin{gathered}
\frac{b_{0 \mid \tau=0}-b_{0}\left(h^{\frac{\alpha}{\gamma-\alpha}}\right)}{h}=O\left(h^{\frac{\gamma(\alpha-1)}{\gamma-\alpha}+\frac{\alpha}{\gamma-\alpha}}\right) \quad \text { in } H^{\infty}, \\
\frac{w_{0 \mid \tau=0}-w_{0}\left(h^{\frac{\alpha}{\gamma-\alpha}}\right)}{h}=O\left(h^{\frac{\gamma(\alpha-1)}{\gamma-\alpha}}\right) \quad \text { in } Y_{(n /(\gamma+1), \infty], 2}^{\infty} .
\end{gathered}
$$

The term $R_{1}^{h}$ satisfies $\left\|R_{1}^{h}\right\|_{H^{s}} \leqslant\left\|b_{0}\right\|_{H^{s+2}} / 2$. Therefore, if $s-2>n / 2+1$, that is, if $s>n / 2+3$ then Proposition 3.5 provides the unique solution $\left(b^{h}, w^{h}\right) \in C([0, T-$

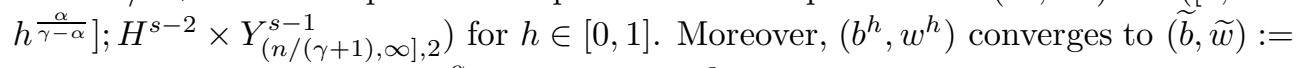
$\left(b^{h}, w^{h}\right)_{\mid h=0}$ in $C\left(\left[0, T-h^{\frac{\alpha}{\gamma-\alpha}}\right] ; H^{s-4} \times Y_{(n /(\gamma+1), \infty], 2}^{s-3}\right)$. It follows from (4.9) that $\lim _{h \rightarrow 0} w_{\mid \tau=0}^{h}=0$ if $\alpha>1$ and $\lim _{h \rightarrow 0} w_{\mid \tau=0}^{h}=-v_{1}$ if $\alpha=1$. Hence, ( $\left.b_{\text {equ }}, w_{\text {equ }}\right)$ solves (4.15)-(4.16).

\section{Supercritical caustic and Supercritical WKB case.}

5.1. Result. In this section, we treat the case $\alpha<1<\gamma$. As presented in Section 4.1, the asymptotic behavior of the solution (1.3)-(1.4) boils down to the asymptotic behavior of the solution to (1.11). By means of Lemma 3.8, we work with $\left(a^{h}, v^{h}\right):=\left(a^{h}, \nabla \phi^{h}\right)$ which solves (4.2)-(4.3).

The main difficulty lies in the fact that the initial data (4.3) is moving at the speed $h^{\frac{\alpha}{\gamma-\alpha}}$ (see Section 2.2). From the expansion (4.1) of $\left(b_{0}, w_{0}\right):=\left(a^{h}, v^{h}\right)_{\mid h=0}$, we deduce that $\left(a^{h}, v^{h}\right)$ contains the terms of order

$$
O\left(h^{\frac{\alpha(\gamma i-1)}{\gamma-\alpha}}\right) \quad \text { and } \quad O\left(h^{\frac{j \alpha \gamma}{\gamma-\alpha}}\right)
$$

for all $i, j \geqslant 0$. Note that some of these orders are less than one if $\alpha<1$. This is the feature of the supercritical WKB case, and the problem comes from this point. Moreover, the above terms interact each other and there appear all the terms whose order is given by the finite combination of $h^{\frac{\alpha(\gamma i-1)}{\gamma-\alpha}}$ and $h^{\frac{j \alpha \gamma}{\gamma-\alpha}}$. Thus, we see that $\left(a^{h}, v^{h}\right)$ contains all the terms whose order is written as

$$
O\left(h^{\frac{\alpha\left(\gamma l_{1}-l_{2}\right)}{\gamma-\alpha}}\right), \quad 0 \leqslant l_{2} \leqslant l_{1} .
$$

For our purpose, we determine all these terms up to $O\left(h^{1}\right)$. Therefore, it is natural to introduce a set $P$ defined by

$$
P:=\left\{\frac{\alpha\left(\gamma l_{1}-l_{2}\right)}{\gamma-\alpha} ; 0 \leqslant l_{2} \leqslant l_{1}, \quad 0 \leqslant \frac{\alpha\left(\gamma l_{1}-l_{2}\right)}{\gamma-\alpha}<1\right\} .
$$


Set $N:=\sharp P-1$, and number the elements of $P$ as $0=p_{0}<p_{1}<\cdots<p_{N}<1$. For any $p_{i_{1}}, p_{i_{2}} \in P$, either $p_{i_{1}}+p_{i_{2}} \in P$ or $p_{i_{1}}+p_{i_{2}} \geqslant 1$ holds. For example, if $\gamma=\sqrt{3}$ and $\alpha=\sqrt{3} / 4$ then, $p_{0}=0$,

$$
\begin{array}{ll}
p_{1}=\frac{\sqrt{3}-1}{3}=\frac{\alpha(\gamma-1)}{\gamma-\alpha}, & p_{2}=\frac{2(\sqrt{3}-1)}{3}=\frac{\alpha(2 \gamma-2)}{\gamma-\alpha}, \\
p_{3}=\frac{\sqrt{3}}{3}=\frac{\alpha \gamma}{\gamma-\alpha}, & p_{4}=\sqrt{3}-1=\frac{\alpha(3 \gamma-3)}{\gamma-\alpha}, \\
p_{5}=\frac{2 \sqrt{3}-1}{3}=\frac{\alpha(2 \gamma-1)}{\gamma-\alpha}, &
\end{array}
$$

and $N=5$; and if $\gamma=2$ and $\alpha=1 / 3$, then $p_{0}=0$,

$$
\begin{aligned}
& p_{1}=\frac{1}{5}=\frac{\alpha(\gamma-1)}{\gamma-\alpha}, \quad p_{2}=\frac{2}{5}=\frac{\alpha(2 \gamma-2)}{\gamma-\alpha}=\frac{\alpha \gamma}{\gamma-\alpha}, \\
& p_{3}=\frac{3}{5}=\frac{\alpha(3 \gamma-3)}{\gamma-\alpha}=\frac{\alpha(2 \gamma-1)}{\gamma-\alpha}, \\
& p_{4}=\frac{4}{5}=\frac{\alpha(4 \gamma-4)}{\gamma-\alpha}=\frac{\alpha(3 \gamma-2)}{\gamma-\alpha}=\frac{2 \alpha \gamma}{\gamma-\alpha},
\end{aligned}
$$

and $N=4$.

To state the result, we also introduce several systems. Let $Q_{1}, Q_{2}$, and $Q_{3}$ be quadratic forms defined in (3.2), (3.3), and (3.4), respectively. Let $a_{l}$ and $v_{l}$ be sequences given in Proposition 4.2. Then, for any $0 \leqslant i \leqslant N$, we introduce

$$
\begin{aligned}
& \left\{\begin{aligned}
\partial_{\tau} b_{i} & =\sum_{p_{j}+p_{k}=p_{i}} Q_{1}\left(b_{p_{j}}, w_{p_{k}}\right), \\
\partial_{\tau} w_{i} & =\sum_{p_{j}+p_{k}=p_{i}}\left(Q_{2}\left(w_{p_{j}}, w_{p_{k}}\right)+\tau^{\gamma-2} Q_{3}\left(b_{p_{j}}, b_{p_{k}}\right)\right),
\end{aligned}\right. \\
& b_{i}(0)= \begin{cases}-a_{l} & \text { if } \exists l \text { such that } p_{i}=\frac{\alpha \gamma l}{\gamma-\alpha}, \\
0 & \text { otherwise, }\end{cases} \\
& w_{i}(0)= \begin{cases}-v_{l^{\prime}} & \text { if } \exists l^{\prime} \text { such that } p_{i}=\frac{\alpha \gamma l^{\prime}-\alpha}{\gamma-\alpha} \\
0 & \text { otherwise. }\end{cases}
\end{aligned}
$$

We also introduce a system for $\left(b_{\text {equ }}, w_{\text {equ }}\right)$

$$
\begin{gathered}
\left\{\begin{aligned}
\partial_{\tau} b_{\text {equ }}= & Q_{1}\left(b_{0}, w_{\text {equ }}\right)+Q_{1}\left(b_{\text {equ }}, w_{0}\right)+\sum_{p_{j}+p_{k}=1} Q_{1}\left(b_{p_{j}}, w_{p_{k}}\right)+\frac{i}{2} \Delta b_{0} \\
\partial_{\tau} w_{\text {equ }}= & Q_{2}\left(w_{0}, w_{\text {equ }}\right)+Q_{2}\left(w_{\text {equ }}, w_{0}\right)+\tau^{\gamma-2}\left(Q_{3}\left(b_{0}, b_{\text {equ }}\right)+Q_{3}\left(b_{\text {equ }}, b_{0}\right)\right) \\
& +\sum_{p_{j}+p_{k}=1}\left(Q_{2}\left(w_{p_{j}}, w_{p_{k}}\right)+\tau^{\gamma-2} Q_{3}\left(b_{p_{j}}, b_{p_{k}}\right)\right)
\end{aligned}\right. \\
b_{\text {equ }}(0)= \begin{cases}-a_{l} & \text { if } \exists l \text { such that } 1=\frac{\alpha \gamma l}{\gamma-\alpha} \\
0 & \text { otherwise }\end{cases} \\
w_{\text {equ }}(0)= \begin{cases}-v_{l^{\prime}} & \text { if } \exists l^{\prime} \text { such that } 1=\frac{\alpha \gamma l^{\prime}-\alpha}{\gamma-\alpha} \\
0 & \text { otherwise },\end{cases}
\end{gathered}
$$


where $\left(b_{0}, w_{0}\right)$ is the solution of (1.13). If there is no pair $(j, k)$ such that $p_{j}+p_{k}=1$, we let $\sum_{p_{j}+p_{k}=1} \equiv 0$. It may happen (see (5.2)).

TheOREm 5.1. Let assumption 1.2 be satisfied. Assume $0<\alpha<1$. Let $P$ be as in (5.1) and $N=\sharp P-1$. Then, there exists an existence time $T>0$ independent of $\varepsilon$. There also exist $\left(b_{j}, \phi_{j}\right) \in C\left([0, T] ; H^{\infty} \times Y_{(n / \gamma, \infty],(n /(\gamma+1), \infty]}^{\infty}\right)$ $(0 \leqslant j \leqslant N)$ such that $\left(b_{i}, w_{i}\right):=\left(b_{i}, \nabla \phi_{i}\right)$ solves $(5.4)-(5.5)$, and $\left(b_{\text {equ }}, \phi_{\text {equ }}\right) \in$ $C\left([0, T] ; H^{\infty} \times Y_{(n / \gamma, \infty],(n /(\gamma+1), \infty]}^{\infty}\right)$ such that $\left(b_{\text {equ }}, w_{\text {equ }}\right):=\left(b_{\text {equ }}, \nabla \phi_{\text {equ }}\right)$ solves (5.6)-(5.7). Moreover, the followings hold:

1. $\phi_{0}(\tau) \asymp \sum_{j=1}^{\infty} \tau^{\gamma j-1} \varphi_{j}$ (in the sense of (1.24)).

2. The solution $u^{\varepsilon}$ to (1.3)-(1.4) satisfies the following asymptotics for all $s \geqslant 0$ : (5.8)

$$
\sup _{t \in\left[0,1-T^{-1} \varepsilon^{\alpha / \gamma}\right]}\left\|\left|J^{\varepsilon}\right|^{s}\left(u^{\varepsilon}(t) e^{-i \Phi^{\varepsilon}(t)}-\frac{1}{(1-t)^{n / 2}} A^{\varepsilon}(t) e^{i \frac{|\cdot|^{2}}{2 \varepsilon(t-1)}}\right)\right\|_{L^{2}} \rightarrow 0
$$

as $\varepsilon \rightarrow 0$ with

$$
\Phi^{\varepsilon}(t, x)=\varepsilon^{\frac{\alpha}{\gamma}-1}\left(\phi_{0}\left(\frac{\varepsilon^{\frac{\alpha}{\gamma}}}{1-t}, \frac{x}{1-t}\right)+\sum_{j=1}^{N} \varepsilon^{\left(1-\frac{\alpha}{\gamma}\right) p_{j}} \phi_{j}\left(\frac{t \varepsilon^{\frac{\alpha}{\gamma}}}{1-t}, \frac{x}{1-t}\right)\right)
$$

and

$$
A^{\varepsilon}(t, x)=b_{0}\left(\frac{\varepsilon^{\frac{\alpha}{\gamma}}}{1-t}, \frac{x}{1-t}\right) \exp \left(i \phi_{\text {equ }}\left(\frac{\varepsilon^{\frac{\alpha}{\gamma}}}{1-t}, \frac{x}{1-t}\right)\right) .
$$

REMARK 5.2. In (5.9), the time variable of $\phi_{j}(j \geqslant 1)$ is not $\varepsilon^{\frac{\alpha}{\gamma}} /(1-t)$ but

$$
\frac{t \varepsilon^{\frac{\alpha}{\gamma}}}{1-t}=\frac{\varepsilon^{\frac{\alpha}{\gamma}}}{1-t}-\varepsilon^{\frac{\alpha}{\gamma}}
$$

Although this variable is not stable on the final layer $1-t=T^{-1} \varepsilon^{\frac{\alpha}{\gamma}}$, this choice is suitable when we work with the well-prepared data (see Section 5.2). Of course, the Taylor expansion

$$
\phi_{j}\left(\frac{t \varepsilon^{\frac{\alpha}{\gamma}}}{1-t}\right)=\sum_{k=0}^{\infty}\left(-\varepsilon^{\frac{\alpha}{\gamma}}\right)^{k}\left(\partial_{t}^{k} \phi_{j}\right)\left(\frac{\varepsilon^{\frac{\alpha}{\gamma}}}{1-t}\right)
$$

will exclude the variable $t \varepsilon^{\frac{\alpha}{\gamma}} /(1-t)$ from $\Phi^{\varepsilon}$, however, we do not pursue this point any more.

REMARK 5.3. Let us classify the phase functions in (5.8) according to the notion in Section 2.2. If $\phi_{i}(0) \not \equiv 0$ (resp. $\left.b_{i}(0) \not \equiv 0\right)$, that is, if there exists a number $l \geqslant 1$ such that $p_{i}=\frac{\alpha \gamma l-\alpha}{\gamma-\alpha}$ (resp. $\left.p_{i}=\frac{\alpha \gamma l}{\gamma-\alpha}\right)$, then $\phi_{i}$ is the correction from phase (resp. correction from amplitude); in particular $\phi_{i}=\phi_{\mathrm{pha}, l}\left(\right.$ resp. $\left.\phi_{i}=\phi_{\mathrm{amp}, l}\right)$. On the other hand, if $\phi_{i}(0) \equiv b_{i}(0) \equiv 0$ then $\phi_{i}$ is the correction from interaction; in particular $\phi_{i}=\phi_{\mathrm{int}, l^{\prime}}$ for some $l^{\prime}$. Notice that the summation in the system (5.4) is decomposed as

$$
\sum_{p_{j}+p_{k}=p_{i}}=\sum_{(j, k)=(i, 0),(0, i)}+\sum_{p_{j}+p_{k}=p_{i}, j k \neq 0} .
$$


The second sum is the interaction term, which is an external force. When $\phi_{i}$ is the correction from interaction, it always has a nonzero interaction term. Otherwise, $\phi_{i} \equiv$ 0 since the system for $\phi_{i}$ is posed with the zero initial condition. There is a possibility that the correction from phase (resp. correction from amplitude) has an interaction term. Indeed, it happens if there is a triplet $j, k, l$ such that $p_{i}=\frac{\alpha \gamma l-\alpha}{\gamma-\alpha}=p_{j}+p_{k}$ (resp. $p_{i}=\frac{\alpha \gamma l}{\gamma-\alpha}=p_{j}+p_{k}$ ) and $j k \neq 0$. In this case, there is a resonance between the correction from phase (resp. correction from amplitude) and the correction from interaction. $\phi_{\text {equ }}$ solving (5.6)-(5.7) is the correction from equation with or without resonance: If $\widetilde{\phi}(0) \not \equiv 0$ (resp. $\widetilde{b}(0) \not \equiv 0)$ then there is a resonance with the correction from phase (resp. correction from amplitude); if $\sum_{p_{j}+p_{k}} \not \equiv 0$ then there is a resonance with the correction from interaction. We note that the resonance among the correction from phase, the correction from interaction, and the correction from equation (resp. the correction from amplitude, the correction from interaction, and the correction from equation) may happen, and that, however, the resonance between the correction from phase and the correction from amplitude never happens because there is no pair $l, l^{\prime}$ such that $\gamma l=\gamma l^{\prime}-1$ if $\gamma>1$.

Proof. As presented in Section 4.1, the asymptotic behavior of the solution (1.3)(1.4) boils down to the asymptotic behavior of the solution to (1.11). By means of Lemma 3.8, we work with $\left(a^{h}, v^{h}\right):=\left(a^{h}, \nabla \phi^{h}\right)$ which solves (4.2)-(4.3).

The existence of $\left(a^{h}, v^{h}\right)$ and the expansion of $\phi_{0}$ are already proven in Propositions 4.1 and 4.2 , respectively. Let $P$ be as in (5.1), $N=\sharp P-1$, and $p_{i} \in P$ $(i=0,1, \ldots, N)$ be such that $\left\{p_{i}\right\}_{i=0}^{N}=P$ and $p_{i}<p_{i+1}$. It suffices to show that $\left(a^{h}, v^{h}\right)$ is expanded as

$$
\begin{aligned}
& a^{h}\left(\tau+h^{\frac{\alpha}{\gamma-\alpha}}\right)=b_{0}\left(\tau+h^{\frac{\alpha}{\gamma-\alpha}}\right)+\sum_{i=1}^{N} h^{p_{i}} b_{i}(\tau)+h b_{\mathrm{equ}}(\tau)+o(h), \\
& v^{h}\left(\tau+h^{\frac{\alpha}{\gamma-\alpha}}\right)=w_{0}\left(\tau+h^{\frac{\alpha}{\gamma-\alpha}}\right)+\sum_{i=1}^{N} h^{p_{i}} w_{i}(\tau)+h w_{\mathrm{equ}}(\tau)+o(h) .
\end{aligned}
$$

Plugging this and

$$
b_{\text {equ }}(\tau)=b_{\text {equ }}\left(\tau+h^{\frac{\alpha}{\gamma-\alpha}}\right)+o(1), \quad w_{\text {equ }}(\tau)=w_{\text {equ }}\left(\tau+h^{\frac{\alpha}{\gamma-\alpha}}\right)+o(1),
$$

to $(1.10)$ and $(1.7)$, we obtain $(5.8)$.

Step 1. We first prove by induction that

$$
\begin{aligned}
& a^{h}\left(\tau+h^{\frac{\alpha}{\gamma-\alpha}}\right)=b_{0}\left(\tau+h^{\frac{\alpha}{\gamma-\alpha}}\right)+\sum_{i=1}^{k} h^{p_{i}} b_{i}(\tau)+o\left(h^{p_{k}}\right), \\
& v^{h}\left(\tau+h^{\frac{\alpha}{\gamma-\alpha}}\right)=w_{0}\left(\tau+h^{\frac{\alpha}{\gamma-\alpha}}\right)+\sum_{i=1}^{k} h^{p_{i}} w_{i}(\tau)+o\left(h^{p_{k}}\right)
\end{aligned}
$$

holds for $k=N$, where $\left(b_{i}, w_{i}\right)$ is a solution of $(5.4)-(5.5)$. One verifies from Proposition 4.1 that (5.11) holds if $k=0$. We put $K \in[1, N]$. We assume for induction that 
(5.11) holds for $k=K-1$, and put

$$
\begin{aligned}
& b_{K}^{h}(\tau)=h^{-p_{K}}\left(a^{h}\left(\tau+h^{\frac{\alpha}{\gamma-\alpha}}\right)-b_{0}\left(\tau+h^{\frac{\alpha}{\gamma-\alpha}}\right)-\sum_{i=1}^{K-1} h^{p_{i}} b_{i}(\tau)\right), \\
& w_{K}^{h}(\tau)=h^{-p_{K}}\left(v^{h}\left(\tau+h^{\frac{\alpha}{\gamma-\alpha}}\right)-w_{0}\left(\tau+h^{\frac{\alpha}{\gamma-\alpha}}\right)-\sum_{i=1}^{K-1} h^{p_{i}} w_{i}(\tau)\right) .
\end{aligned}
$$

By the equation for $\left(a^{h}, v^{h}\right)$, we see that $\left(b_{K}^{h}, w_{K}^{h}\right)$ solves

$$
\left\{\begin{aligned}
\partial_{\tau} b_{K}^{h}= & h^{p_{K}} Q_{1}\left(b_{K}^{h}, w_{K}^{h}\right)+Q_{1}\left(B_{1}^{h}, w_{K}^{h}\right)+Q_{1}\left(b_{K}^{h}, W_{1}^{k}\right)+R_{1}^{h}+\frac{i}{2} h \Delta b_{K}^{h}, \\
\partial_{\tau} w_{K}^{h}= & h^{p_{K}} Q_{2}\left(w_{K}^{h}, w_{K}^{h}\right)+Q_{2}\left(W_{2}^{h}, w_{K}^{h}\right)+Q_{2}\left(w_{K}^{h}, W_{2}^{k}\right) \\
& +\left(\tau+h^{\frac{\alpha}{\gamma-\alpha}}\right)^{\gamma-2}\left(h^{p_{K}} Q_{3}\left(b_{K}^{h}, b_{K}^{h}\right)+Q_{3}\left(B_{2}^{h}, b_{K}^{h}\right)+Q_{3}\left(b_{K}^{h}, B_{2}^{k}\right)\right) \\
& +R_{2}^{h}, \\
b_{K}^{h}(0)= & h^{-p_{K}}\left(a_{0}-b_{0}\left(h^{\frac{\alpha}{\gamma-\alpha}}\right)-\sum_{i=1}^{K-1} h^{p_{i}} b_{i}(0)\right), \\
w_{K}^{h}(0)= & h^{-p_{K}}\left(-w_{0}\left(h^{\frac{\alpha}{\gamma-\alpha}}\right)-\sum_{i=1}^{K-1} h^{p_{i}} w_{i}(0)\right),
\end{aligned}\right.
$$

where

$$
\begin{gathered}
B_{1}^{h}=B_{2}^{h}=\sum_{i=0}^{K-1} h^{p_{i}} b_{i} \rightarrow b_{0}(t), \\
W_{1}^{h}=W_{2}^{h}=\sum_{i=0}^{K-1} h^{p_{i}} w_{i} \rightarrow w_{0}(t), \\
R_{1}^{h}=\sum_{p_{l}<K, p_{k}<K, p_{l}+p_{k}=p_{K}} Q_{1}\left(b_{p_{l}}, w_{p_{k}}\right) \\
+\sum_{p_{l}<K, p_{k}<K, p_{l}+p_{k}>p_{K}} h^{p_{l}+p_{k}-p_{K}} Q_{1}\left(b_{p_{l}}, w_{p_{k}}\right) \\
+\frac{i h^{1-p_{K}}}{2} \sum_{i=0}^{K-1} h^{p_{i}} \Delta b_{i} \\
\sum_{p_{l}<K, p_{k}<K, p_{l}+p_{k}=p_{K}} Q_{1}\left(b_{p_{l}}, w_{p_{k}}\right),
\end{gathered}
$$

and

$$
\begin{aligned}
R_{2}^{h}= & \sum_{p_{l}<K, p_{k}<K, p_{l}+p_{k}=p_{K}}\left(Q_{2}\left(w_{p_{l}}, w_{p_{k}}\right)+\left(t+h^{\frac{\alpha}{\gamma-\alpha}}\right)^{\gamma-2} Q_{3}\left(b_{p_{l}}, b_{p_{k}}\right)\right) \\
& +\sum_{p_{l}<K, p_{k}<K, p_{l}+p_{k}>p_{K}} h^{p_{l}+p_{k}-p_{K}} Q_{2}\left(w_{p_{l}}, w_{p_{k}}\right) \\
& +\sum_{p_{l}<K, p_{k}<K, p_{l}+p_{k}>p_{K}} h^{p_{l}+p_{k}-p_{K}}\left(t+h^{\frac{\alpha}{\gamma-\alpha}}\right)^{\gamma-2} Q_{3}\left(b_{p_{l}}, b_{p_{k}}\right) \\
& \rightarrow \sum_{p_{l}<K, p_{k}<K, p_{l}+p_{k}=p_{K}}\left(Q_{2}\left(w_{p_{l}}, w_{p_{k}}\right)+t^{\gamma-2} Q_{3}\left(b_{p_{l}}, b_{p_{k}}\right)\right) .
\end{aligned}
$$


Moreover, applying the time expansion of $\left(b_{0}, w_{0}\right)$, we deduce that $\left(b_{K}^{h}(0), w_{K}^{h}(0)\right)$ is uniformly bounded and that, as $h \rightarrow 0$,

$$
\begin{aligned}
b_{K}^{h}(0) & =-h^{-p_{K}}\left(b_{0}\left(h^{\frac{\alpha}{\gamma-\alpha}}\right)-\sum_{\left\{j ; \frac{\alpha \gamma j}{\gamma-\alpha}<p_{K}\right\}} h^{\frac{\alpha \gamma j}{\gamma-\alpha}} a_{j}\right) \\
& \rightarrow \begin{cases}-a_{j^{\prime}} & \text { if } \exists j^{\prime} \text { such that } p_{K}=\frac{\alpha \gamma j^{\prime}}{\gamma-\alpha}, \\
0 & \text { otherwise, }\end{cases} \\
w_{K}^{h}(0) & =-h^{-p_{K}}\left(\begin{array}{ll}
w_{0}\left(h^{\frac{\alpha}{\gamma-\alpha}}\right)-\sum_{\left\{j ; \frac{\alpha(\gamma j-1)}{\gamma-\alpha}<p_{K}\right\}} h^{\frac{\alpha(\gamma j-1)}{\gamma-\alpha}} v_{j}
\end{array}\right) \\
& \rightarrow \begin{cases}-v_{j^{\prime \prime}} & \text { if } \exists j^{\prime \prime} \text { such that } p_{K}=\frac{\alpha\left(\gamma j^{\prime \prime}-1\right)}{\gamma-\alpha}, \\
0 & \text { otherwise. }\end{cases}
\end{aligned}
$$

Note that either $b_{K}^{h}(0) \rightarrow 0$ or $w_{K}^{h}(0) \rightarrow 0$ holds for all $K$ since $\gamma>1$ implies there is no pair $\left(j, j^{\prime}\right)$ such that $\gamma j=\gamma j^{\prime}-1$. Therefore, we apply Proposition 3.5 to obtain the solution $\left(b_{K}^{h}, w_{K}^{h}\right)$. Put $\left(b_{K}, w_{K}\right):=\left(b_{K}^{h}, w_{K}^{h}\right)_{\mid h=0}$. Then, it solves (5.4)-(5.5), and so (5.11) holds for $k=K$. By induction (5.11) holds for $k=N$.

Step 2. Mimicking the argument in Step 1, we construct $\left(b_{\text {equ }}, w_{\text {equ }}\right)$ such that

$$
\begin{aligned}
& a^{h}\left(\tau+h^{\frac{\alpha}{\gamma-\alpha}}\right)=b_{0}\left(\tau+h^{\frac{\alpha}{\gamma-\alpha}}\right)+\sum_{i=1}^{N} h^{p_{i}} b_{i}(\tau)+h b_{\text {equ }}(\tau)+o(h), \\
& v^{h}\left(\tau+h^{\frac{\alpha}{\gamma-\alpha}}\right)=w_{0}\left(\tau+h^{\frac{\alpha}{\gamma-\alpha}}\right)+\sum_{i=1}^{N} h^{p_{i}} w_{i}(\tau)+h w_{\text {equ }}(\tau)+o(h)
\end{aligned}
$$

hold. Notice that $\left(b_{\text {equ }}, w_{\text {equ }}\right)$ solves

$$
\begin{aligned}
& \partial_{\tau} b_{\text {equ }}= Q_{1}\left(b_{0}, w_{\text {equ }}\right)+Q_{1}\left(b_{\text {equ }}, w_{0}\right)+\sum_{p_{j}+p_{k}=1} Q_{1}\left(b_{p_{j}}, w_{p_{k}}\right)+\frac{i}{2} \Delta b_{0}, \\
& \partial_{\tau} w_{\text {equ }}= Q_{2}\left(w_{0}, w_{\text {equ }}\right)+Q_{2}\left(w_{\text {equ }}, w_{0}\right)+\tau^{\gamma-2}\left(Q_{3}\left(b_{0}, b_{\text {equ }}\right)+Q_{3}\left(b_{\text {equ }}, b_{0}\right)\right) \\
&+\sum_{p_{j}+p_{k}=1}\left(Q_{2}\left(w_{p_{j}}, w_{p_{k}}\right)+\tau^{\gamma-2} Q_{3}\left(b_{p_{j}}, b_{p_{k}}\right)\right), \\
& \widetilde{b}(0)=\left\{\begin{array}{ll}
-a_{l} & \text { if } 1=\frac{\alpha \gamma l}{\gamma-\alpha} \\
0 & \text { otherwise },
\end{array} \quad \widetilde{w}(0)= \begin{cases}-v_{l^{\prime}} & \text { if } 1=\frac{\alpha \gamma l^{\prime}-\alpha}{\gamma-\alpha} \exists l^{\prime}, \\
0 & \text { otherwise. }\end{cases} \right.
\end{aligned}
$$

REMARK 5.4. By a similar proof, we obtain higher order approximation. Let $0<\alpha<\gamma$ and $\gamma>1$. We modify the set $P$ defined by (5.1) as

$$
P^{\prime}:=\left\{l+\frac{\alpha \gamma m}{\gamma-\alpha}+\frac{\alpha(\gamma-1) n}{\gamma-\alpha} ; \quad l, m, n \geqslant 0\right\}
$$


and number the elements of $P^{\prime}$ as $0=p_{0}<p_{1}<\cdots<p_{k}<\ldots$. Then, we have, for all $k$,

$$
\begin{aligned}
& a^{h}\left(\tau+h^{\frac{\alpha}{\gamma-\alpha}}\right)=b_{0}\left(\tau+h^{\frac{\alpha}{\gamma-\alpha}}\right)+\sum_{i=1}^{k} h^{p_{i}} b_{i}(\tau)+o\left(h^{p_{k}}\right), \\
& \phi^{h}\left(\tau+h^{\frac{\alpha}{\gamma-\alpha}}\right)=\phi_{0}\left(\tau+h^{\frac{\alpha}{\gamma-\alpha}}\right)+\sum_{i=1}^{k} h^{p_{i}} \phi_{i}(\tau)+o\left(h^{p_{k}}\right) .
\end{aligned}
$$

Plugging this to (1.10) and (1.7), we obtain higher order approximation of the original solution. It is important to note that the (5.12) has the same form in the both $\alpha \geqslant 1$ and $\alpha<1$ case. When we concerned with higher order WKB-type approximation, we need four kinds of correction terms even if $\alpha \geqslant 1$. From this respect, the supercritical WKB case $\alpha<1$ can be characterized as the the special case $p_{1}<1$.

5.2. Well-prepared data and general data. We conclude this section with some remarks about the well-prepared data. By the semiclassical conformal transform (1.7) and Grenier's transform (1.10), the leading order WKB-type approximation of the original solution $u^{\varepsilon}$ to (1.3)-(1.4) is reduced to the approximation of the solution $\left(a^{h}, \phi^{h}\right)$ to

$$
\left\{\begin{array}{l}
\partial_{\tau} a^{h}+\nabla \phi^{h} \cdot \nabla a^{h}+\frac{1}{2} a^{h} \Delta \phi^{h}=i \frac{h}{2} \Delta a^{h}, \\
\partial_{\tau} \phi^{h}+\frac{1}{2}\left|\nabla \phi^{h}\right|^{2}+\lambda \tau^{\gamma-2}\left(|y|^{-\gamma} *\left|a^{h}\right|^{2}\right)=0
\end{array}\right.
$$

with

$$
a_{\mid \tau=h \frac{\alpha}{\gamma-\alpha}}^{h}=a_{0}, \quad \phi_{\mid \tau=h}^{h} \frac{\alpha}{\gamma-\alpha}=0,
$$

up to order $O\left(h^{1}\right)$. Note that (5.13)-(5.14) and (1.11) are the same. As shown in Proposition 4.1 , there exists a limit $\left(b_{0}, \phi_{0}\right):=\left(a^{h}, \phi^{h}\right)_{\mid h=0}$ which solves (1.13). Now, we consider the distances

$$
d_{a}^{h}(t):=a^{h}(t)-b_{0}(t), \quad d_{\phi}^{h}(t):=\phi^{h}(t)-\phi_{0}(t) .
$$

If these distances are order $o\left(h^{1}\right)$ then we immediately obtain the WKB-type approximation $b_{0} \exp \left(i \varepsilon^{\frac{\alpha}{\gamma}-1} \phi_{0}\right)$ of $u^{\varepsilon}$ (recall that $\left.h=\varepsilon^{1-\frac{\alpha}{\gamma}}\right)$. However, unfortunately, the following two respects prevent us: The first one is the $h$-dependence of the equation (5.13), and the second one is the $h$-dependence of the initial time (5.14). The first problem is handled by employing the correction term $\left(b_{\text {equ }}, \phi_{\text {equ }}\right)$ solving $(1.18)$ and, therefore we discuss about the initial data in the followings.

The given initial data (5.14) is written as

$$
d_{a}^{h}\left(h^{\frac{\alpha}{\gamma-\alpha}}\right):=a_{0}-b_{0}\left(h^{\frac{\alpha}{\gamma-\alpha}}\right), \quad \quad d_{\phi}^{h}\left(h^{\frac{\alpha}{\gamma-\alpha}}\right):=-\phi_{0}\left(h^{\frac{\alpha}{\gamma-\alpha}}\right) .
$$

The main difficulty in the case $\alpha \leqslant 1$ is the fact that these terms become larger than $O\left(h^{1}\right)$ as $h \rightarrow 0$. The simplest way to overcome this difficulty is to modify the initial data (5.14) into

$$
a_{\mid \tau=h}^{h} \frac{\alpha}{\gamma-\alpha}=b_{0}\left(h^{\frac{\alpha}{\gamma-\alpha}}\right), \quad \phi_{\mid \tau=h}^{h}, \quad \frac{\alpha}{\gamma-\alpha}=\phi_{0}\left(h^{\frac{\alpha}{\gamma-\alpha}}\right),
$$


which ensures $d_{a}^{h}\left(h^{\frac{\alpha}{\gamma-\alpha}}\right) \equiv d_{\phi}^{h}\left(h^{\frac{\alpha}{\gamma-\alpha}}\right) \equiv 0$. Note that (5.13) with (5.15) is the same as (1.14). Back to the transform (1.7), this initial data corresponds to the well-prepared data

$$
u_{\mid t=0}^{\varepsilon}(x)=b_{0}\left(\varepsilon^{\frac{\alpha}{\gamma}}, x\right) e^{-i \frac{|x|^{2}}{2 \varepsilon}} \exp \left(i \varepsilon^{\frac{\alpha}{\gamma}-1} \phi_{0}\left(\varepsilon^{\frac{\alpha}{\gamma}}, x\right)\right)
$$

This initial condition is rather natural in the supercritical case $\alpha<1$, and the original initial condition in (1.4) is a kind of constraint (see, Section 2.2). If we use this wellprepared data, we do not have to consider any correction term other than $\left(b_{\text {equ }}, \phi_{\text {equ }}\right)$ and, for all $0<\alpha<\gamma$, it holds that

$$
\begin{aligned}
& a^{h}(\tau)=b_{0}(\tau)+h b_{\text {equ }}(\tau)+o(h), \\
& \phi^{h}(\tau)=\phi_{0}(\tau)+h \phi_{\text {equ }}(\tau)+o(h)
\end{aligned}
$$

with $\left(b_{0}, \phi_{0}\right)$ and $\left(b_{\text {equ }}, \phi_{\text {equ }}\right)$ solving (1.13) and (1.18)-(1.19), respectively, and so that the asymptotic behavior of the solution $u^{\varepsilon}(t, x)$ to $(1.3)-(1.6)$ is given by

$$
b_{0}\left(\frac{\varepsilon^{\frac{\alpha}{\gamma}}}{1-t}, \frac{x}{1-t}\right) \exp \left(i \phi_{\text {equ }}\left(\frac{\varepsilon^{\frac{\alpha}{\gamma}}}{1-t}, \frac{x}{1-t}\right)+i \varepsilon^{\frac{\alpha}{\gamma}-1} \phi_{0}\left(\frac{\varepsilon^{\frac{\alpha}{\gamma}}}{1-t}, \frac{x}{1-t}\right)\right) .
$$

This approximate solution is the same one as in the case $\alpha>1$. We still need $O\left(h^{1}\right)$ correction term $\left(b_{\text {equ }}, \phi_{\text {equ }}\right)$ because it comes from the $h^{1}$-dependence of the equation which $\left(a^{h}, \phi^{h}\right)$ solves.

In Theorems 1.3 and 5.1, we took another way. By the expansion of $\left(b_{0}, \phi_{0}\right)$ around $\tau=0$, there exist nonnegative integers $k_{1}, k_{2}$ depending on $\alpha$ and $\gamma$ such that

$$
\begin{aligned}
& d_{a}^{h}\left(h^{\frac{\alpha}{\gamma-\alpha}}\right)=a_{0}-b_{0}\left(h^{\frac{\alpha}{\gamma-\alpha}}\right)=-\sum_{j=1}^{k_{1}} h^{\frac{\alpha \gamma j}{\gamma-\alpha}} a_{j}+o\left(h^{1}\right), \\
& d_{\phi}^{h}\left(h^{\frac{\alpha}{\gamma-\alpha}}\right)=-\phi_{0}\left(h^{\frac{\alpha}{\gamma-\alpha}}\right)=-\sum_{j=1}^{k_{2}} h^{\frac{\alpha(\gamma j-1)}{\gamma-\alpha}} \varphi_{j}+o\left(h^{1}\right) .
\end{aligned}
$$

We subtract the main part $-\sum_{j=1}^{k_{1}} h^{\frac{\alpha \gamma j}{\gamma-\alpha}} a_{j}$ and $-\sum_{j=1}^{k_{2}} h^{\frac{\alpha(\gamma j-1)}{\gamma-\alpha}} \varphi_{j}$ by constructing appropriate correction terms (correction from amplitude and correction from phase, respectively). Indeed, if we let the correction terms $\left(b_{j}, \phi_{j}\right)(0 \leqslant j \leqslant N)$ and $\left(b_{\text {equ }}, \phi_{\text {equ }}\right)$ be defined as in Theorem 5.1 then, at the initial time $\tau=h^{\frac{\alpha}{\gamma-\alpha}}$, it holds that

$$
\begin{aligned}
& \left(a^{h}(\tau)-b_{0}(\tau)-\sum_{j=1}^{N} h^{p_{j}} b_{j}\left(\tau-h^{\frac{\alpha}{\gamma-\alpha}}\right)-h b_{\text {equ }}(\tau)\right)_{\mid \tau=h^{\frac{\alpha}{\gamma-\alpha}}} \\
& =-\left(b_{0}\left(h^{\frac{\alpha}{\gamma-\alpha}}\right)-\sum_{l \in\left\{l \geqslant 0 ; \frac{\alpha \gamma l}{\gamma-\alpha} \leqslant 1\right\}} h^{\frac{\alpha \gamma l}{\gamma-\alpha}} a_{l}\right)+h\left(b_{\text {equ }}(0)-b_{\text {equ }}\left(h^{\frac{\alpha}{\gamma-\alpha}}\right)\right) \\
& =o\left(h^{1}\right),
\end{aligned}
$$




$$
\begin{aligned}
& \left(\phi^{h}(\tau)-\phi_{0}(\tau)-\sum_{j=1}^{N} h^{p_{j}} \phi_{j}\left(\tau-h^{\frac{\alpha}{\gamma-\alpha}}\right)-h \phi_{\text {equ }}(t)\right)_{\mid \tau=h \frac{\alpha}{\gamma-\alpha}} \\
& =-\left(\phi_{0}\left(h^{\frac{\alpha}{\gamma-\alpha}}\right)-\sum_{l \in\left\{l \geqslant 1 ; \frac{\alpha(\gamma l-1)}{\gamma-\alpha} \leqslant 1\right\}} h^{\frac{\alpha(\gamma l-1)}{\gamma-\alpha}} \varphi_{l}\right)+h\left(\phi_{\text {equ }}(0)-\phi_{\text {equ }}\left(h^{\frac{\alpha}{\gamma-\alpha}}\right)\right) \\
& =o\left(h^{1}\right) .
\end{aligned}
$$

It is important to note that the time variable of $\left(b_{j}, \phi_{j}\right)(1 \leqslant j \leqslant N)$ is not $\tau$ but $\tau-h^{\frac{\alpha}{\gamma-\alpha}}$. This choice is the key for the above cancellation. By the transform (1.7), the time variable of $\phi_{j}(1 \leqslant j \leqslant N)$ in the definition (5.9) of $\Phi^{\varepsilon}(t)$ should be given by

$$
\frac{\varepsilon^{\frac{\alpha}{\gamma}}}{1-t}-\varepsilon^{\frac{\alpha}{\gamma}}=\frac{t \varepsilon^{\frac{\alpha}{\gamma}}}{1-t}
$$

These correction terms allow us to work with general data.

6. Proofs of Theorems 1.5 and 1.6. Recall that the system we consider is

$$
\left\{\begin{array}{l}
\partial_{\tau}\left(\rho r^{n-1}\right)+\partial_{r}\left(\rho v r^{n-1}\right)=0 \\
\partial_{\tau} v+v \partial_{r} v-\lambda \tau^{n-4} \partial_{r} V_{\mathrm{p}}=0 \\
\partial_{r}\left(r^{n-1} \partial_{r} V_{\mathrm{p}}\right)=\rho r^{n-1} \\
\rho_{\mid \tau=0}=\left|a_{0}\right|^{2}, \quad v_{\mid \tau=0}=0
\end{array}\right.
$$

where $r=|x|$. We introduce the "mass" $m$ and the "mean mass" $M$

$$
m(\tau, r):=M(\tau, r) r^{n}:=r^{n-1} \partial_{r} \Phi(\tau, r)=\int_{0}^{r} \rho(\tau, s) s^{n-1} d s .
$$

We also set $m_{0}(r):=m(0, r)$ and $M_{0}(r):=M(0, r)$. Combining the first and the third equations of (1.26), we obtain

$$
\partial_{\tau} m+v \partial_{r} m=0
$$

where we have used $\left(\rho v r^{n-1}\right)_{\mid r=0}=0$. To solve this equation we also introduce the characteristic curve $X(\tau, R)$ :

$$
\frac{d X}{d \tau}=v(\tau, X(\tau, R)), \quad X(0, R)=R
$$

Denoting differentiation along this characteristic curve by $1:=d / d \tau$, the mass equation (6.1) and the second equation of (1.26) yield

$$
\begin{aligned}
& m^{\prime}=0, \\
& v^{\prime}=\lambda \tau^{n-4} \frac{m}{X^{n-1}}, \\
& X^{\prime}=v .
\end{aligned}
$$

We solve this system with the initial data

$$
(X, m, v)_{\mid \tau=0}=\left(R, m_{0}(R), 0\right),
$$


where $R \geq 0$ parameterizes the initial location.

By (6.2), the mass $m$ remains constant along the characteristics, that is, $m(\tau, X(\tau, R))=m_{0}(R)$. Therefore, (6.3) and (6.4) yield

$$
X^{\prime \prime}=\frac{\lambda m_{0}(R) \tau^{n-4}}{X^{n-1}}, \quad X(0, R)=R, \quad X^{\prime}(0, R)=0 .
$$

This equation is studied also in [29].

Proof of Theorem 1.5. It suffices to show that $X(\tau, R) \leqslant 0$ holds for some $R>0$ and $\tau \leqslant T^{*}$. This argument is similar to that for the blow-up for the nonlinear Schrödinger equation by Glassey [17]. Since $\lambda<0$, we see from (6.5) that $X^{\prime \prime} \leqslant 0$, and so that $X^{\prime} \leqslant X^{\prime}(0)=0$ and $X \leqslant X(0)=R$ for all $\tau \geqslant 0$. Therefore, again by (6.5), we verify that

$$
X^{\prime \prime}(\tau, R) \leqslant-\frac{|\lambda| m_{0} \tau^{n-4}}{R^{n-1}}=-|\lambda| R M_{0}(R) \tau^{n-4} .
$$

Note that $M_{0}(R)>0$ for some $R$ provided $a_{0}$ is not identically zero. Now, fix such $R$. Integrating twice with respect to time, we obtain

$$
X(\tau, R) \leqslant R-\frac{|\lambda| R M_{0}(R)}{(n-2)(n-3)} \tau^{n-2},
$$

which yields $X(\tau, R) \leqslant 0$ for large time. The critical time is not greater than

$$
T^{*}=\left(\frac{(n-2)(n-3)}{|\lambda| \sup _{R \geqslant 0} M_{0}(R)}\right)^{\frac{1}{n-2}}
$$

since $R$ is arbitrary.

Proof of Theorem 1.6. In order to clarify the necessary and sufficient condition, we repeat the proof in [12]. We first note that $\partial X / \partial R(0, R)=1$, and that the solution is global if and only if $\partial X / \partial R(t, R)>0$ for all $\tau \geqslant 0$ and $R \geqslant 0$.

By (6.5), we have

$$
X^{\prime \prime}=\frac{\lambda m_{0}}{X^{3}} .
$$

We multiply this by $X^{\prime}$ and integrate in time to obtain

$$
\left(X^{\prime}\right)^{2}=0^{2}+\frac{\lambda m_{0}}{R^{2}}-\frac{\lambda m_{0}}{X^{2}}=\frac{\lambda m_{0}}{R^{2}}-X X^{\prime \prime},
$$

where we have used (6.5) again. This yields

$$
\left(X^{2}\right)^{\prime \prime}=2\left(X^{\prime}\right)^{2}+2 X X^{\prime \prime}=\frac{2 \lambda m_{0}}{R^{2}} .
$$

Then, integration twice gives

$$
X^{2}=R^{2}+\frac{\lambda m_{0}}{R^{2}} t^{2}
$$

Since $X \geqslant R>0$ from (6.5) and $X^{\prime}(0)=0$, we see

$$
X(\tau, R)=\sqrt{R^{2}+\frac{\lambda m_{0}}{R^{2}} \tau^{2}} .
$$


An explicit calculation shows that

$$
\frac{\partial X}{\partial R}(\tau, R)=\frac{1+\lambda\left(\frac{1}{2}\left|a_{0}\right|^{2}-M_{0}\right) \tau^{2}}{\sqrt{1+\lambda M_{0} \tau^{2}}}
$$

where $m_{0}=M_{0} R^{4}=\int_{0}^{R}\left|a_{0}\right|^{2} s^{3} d s$ by definition. Note that $\lim _{R \rightarrow 0} M_{0}=\left|a_{0}(0)\right|^{2} / 4$ since $a_{0}$ is continuous. Hence, $\partial X / \partial R(t, R)>0$ holds for all $\tau \geqslant 0$ and $R \geqslant 0$ if and only if $\frac{1}{2}\left|a_{0}\right|^{2}-M_{0} \geqslant 0$ for all $R>0$, that is,

$$
\left|a_{0}(R)\right|^{2} \geqslant \frac{2}{R^{4}} \int_{0}^{R}\left|a_{0}(s)\right|^{2} s^{3} d s
$$

for all $R>0$. Moreover, the critical time is given by

$$
\tau_{c}=\left(\frac{2}{\lambda \max _{r>0}\left(2 M_{0}(r)-\left|a_{0}(r)\right|^{2}\right)}\right)^{\frac{1}{2}} .
$$

The condition (6.7) can be written as

$$
\partial_{R}\left(\frac{m_{0}(R)}{R^{2}}\right) \geqslant 0
$$

We take $R_{0}$ so that $m_{0}\left(R_{0}\right)>0$. Then, it holds only if

$$
m_{0}(R) \geqslant \frac{m_{0}\left(R_{0}\right)}{R_{0}^{2}} R^{2}
$$

for all $R \geqslant R_{0}$, which fails if $\lim _{R \rightarrow \infty} m_{0}(R)<\infty$, that is, if $a_{0} \in L^{2}\left(\mathbb{R}^{4}\right)$.

Remark 6.1. If $n=4$ then (1.26) becomes an autonomous system. Now we consider the classical solution of autonomous model

$$
\left\{\begin{array}{l}
\partial_{\tau}\left(\rho r^{n-1}\right)+\partial_{r}\left(\rho v r^{n-1}\right)=0 \\
\partial_{\tau} v+v \partial_{r} v-\lambda \partial_{r} V_{\mathrm{p}}=0 \\
\partial_{r}\left(r^{n-1} \partial_{r} V_{\mathrm{p}}\right)=\rho r^{n-1} \\
\rho_{\mid \tau=0}=\rho_{0}, \quad v_{\mid \tau=0}=v_{0}
\end{array}\right.
$$

where $(\tau, r) \in \mathbb{R}_{+} \times \mathbb{R}_{+}$. It is shown in [25] that, under the assumption that $n \geqslant 3$, $\rho_{0} \in L^{1}\left((0, \infty), r^{n-1} d r\right), v_{0}(0)=0$, and $v_{0}(r) \rightarrow 0$ as $r \rightarrow \infty$, the corresponding solution is global if and only if $\lambda<0$ and

$$
v_{0}(r)=\sqrt{\frac{2|\lambda|}{(n-2) r^{n-2}} \int_{0}^{r} \rho_{0}(s) s^{n-1} d s .}
$$

REMARK 6.2. The function $\Gamma=\partial X / \partial R$ is called the indicator function. As in the above proof, the solution blows up if and only if $\Gamma$ takes non-positive value. Moreover, the solution is given by

$$
\begin{aligned}
\rho(t, X(t, R)) & =\frac{\rho_{0}(R) R^{n-1}}{X^{n-1}(t, R) \Gamma(t, R)}, \\
v(t, X(t, R)) & =\frac{d X}{d t}(t, R) .
\end{aligned}
$$

Example 1.7 is easily checked by this form since the characteristic curve $X$ is given explicitly by (6.6) in the case of $\lambda<0$ and $N=4$. 
Acknowledgments. The author expresses his deep gratitude to Professor Rémi Carles for his reading an early version of the paper and fruitful discussions in Montpellier. Deep appreciation goes to Professor Yoshio Tsutsumi for his valuable advice and constant encouragement. This research progressed during the author's stay in Orsay. The author is grateful to people in department of mathematics, University of Paris 11 for their kind hospitality. This research is supported by Grant-in-Aid for JSPS fellows.

\section{REFERENCES}

[1] T. Alazard and R. Carles, Semi-classical limit of Schrödinger-Poisson equations in space dimension $n \geqslant 3$, J. Differential Equations, 233:1 (2007), pp. 241-275.

[2] $\longrightarrow$ Supercritical geometric optics for nonlinear Schrödinger equations, Arch. Ration. Mech. Anal., 194:1 (2009), pp. 315-347.

[3] — WKB analysis for the Gross-Pitaevskii equation with non-trivial boundary conditions at infinity, Ann. Inst. H. Poincare Anal. Non Lineaire, 26:3 (2009), pp. 959-977.

[4] R. CARLES, Geometric optics with caustic crossing for some nonlinear Schrödinger equations, Indiana Univ. Math. J., 49:2 (2000), pp. 475-551.

[5] - Geometric optics and long range scattering for one-dimensional nonlinear Schrödinger equations, Comm. Math. Phys., 220:1 (2001), pp. 41-67.

[6] Cascade of phase shifts for nonlinear Schrödinger equations, J. Hyperbolic Differ. Equ., 4:2 (2007), pp. 207-231.

[7] _ Semi-classical analysis for nonlinear Schrödinger equations, World Scientific Publishing Co. Pte. Ltd., Hackensack, NJ, 2008.

[8] R. Carles And D. Lannes, Focusing at a point with caustic crossing for a class of nonlinear equations, 2nd France-Tunisia meeting (2003).

[9] R. Carles and S. Masaki, Semiclassical analysis for Hartree equations, Asymptotic Analysis, 58:4 (2008), pp. 211-227.

[10] R. Carles, N. J. Mauser, and H. P. Stimming, (semi)classical limit of the Hartree equation with harmonic potential, SIAM J. Appl. Math, 66:1 (2005), pp. 29-56.

[11] D. Chiron and F. Rousset, Geometric optics and boundary layers for Nonlinear-Schrödinger Equations, Comm. Math. Phys., 288:2 (2009), pp. 503-546.

[12] S. Engelberg, H. Liu, And E. TADmor, Critical thresholds in Euler-Poisson equations, Indiana Univ. Math. J., 50 (2001), no. Special Issue, 109-157, Dedicated to Professors Ciprian Foias and Roger Temam (Bloomington, IN, 2000).

[13] C. Gallo, Schrödinger group on Zhidkov spaces, Adv. Differential Equations, 9:5-6 (2004), pp. 509-538.

[14] I. Gasser, C.-K. Lin, and P. A. Markowich, A review of dispersive limits of (non)linear Schrödinger-type equations, Taiwanese J. Math., 4:4 (2000), pp. 501-529.

[15] P. GÉRARD, Remarques sur l'analyse semi-classique de l'équation de Schrödinger non linéaire, Séminaire sur les Équations aux Dérivées Partielles, 1992-1993, École Polytech., Palaiseau, 1993, pp. Exp. No. XIII, 13.

[16] - The Cauchy problem for the Gross-Pitaevskii equation, Ann. Inst. H. Poincaré Anal. Non Linéaire, 23:5 (2006), pp. 765-779.

[17] R. T. Glassey, On the blowing up of solutions to the Cauchy problem for nonlinear Schrödinger equations, J. Math. Phys., 18:9 (1977), pp. 1794-1797.

[18] E. Grenier, Semiclassical limit of the nonlinear Schrödinger equation in small time, Proc. Amer. Math. Soc., 126:2 (1998), pp. 523-530.

[19] L. Hörmander, The analysis of linear partial differential operators. I, second ed., Springer Study Edition, Springer-Verlag, Berlin, 1990, Distribution theory and Fourier analysis.

[20] J. Hunter And J. Keller, Caustics of nonlinear waves, Wave motion, 9 (1987), pp. 429-443.

[21] H. Li AND C.-K. Lin, Semiclassical limit and well-posedness of nonlinear Schrödinger-Poisson systems, Electron. J. Differential Equations (2003), No. 93, 17 pp. (electronic).

[22] F. Lin and P. Zhang, Semiclassical limit of the Gross-Pitaevskii equation in an exterior domain, Arch. Ration. Mech. Anal., 179:1 (2006), pp. 79-107.

[23] H. Liu AND E. TADMOR, Semiclassical limit of the nonlinear Schrödinger-Poisson equation with subcritical initial data, Methods Appl. Anal., 9:4 (2002), pp. 517-531.

[24] T. Makino and B. Perthame, Sur les solutions à symétrie sphérique de l'équation d'EulerPoisson pour l'évolution d'étoiles gazeuses, Japan J. Appl. Math., 7:1 (1990), pp. 165-170. 
[25] S. MASAKI, Remarks on global existence of classical solution to multi-dimensional compressible Euler-Poisson equations with geometrical symmetry, RIMS Kokyuroku Bessatsu, to appear.

[26] Semi-classical analysis for Hartree equations in some supercritical cases, Ann. Henri Poincaré, 8:6 (2007), pp. 1037-1069.

[27] Semi-classical analysis of the Hartree equation around and before the caustic, Adv. Stud. in Pure Math., 47:1 (2007), pp. 217-236.

[28] B. Perthame, Nonexistence of global solutions to Euler-Poisson equations for repulsive forces, Japan J. Appl. Math., 7:2 (1990), pp. 363-367.

[29] I. Tsuкамото, On asymptotic behavior of positive solutions of $x^{\prime \prime}=-t^{\alpha \lambda-2} x^{1+\alpha}$ with $\alpha<0$ and $-1<\lambda<0$, preprint, 2009.

[30] P. Zhang, Wigner measure and the semiclassical limit of Schrödinger-Poisson equations, SIAM J. Math. Anal., 34:3 (2002), pp. 700-718 (electronic).

[31] P. E. Zhidkov, The Cauchy problem for a nonlinear Schrödinger equation, JINR Commun., P5-87-373, Dubna (1987), (in Russian). 